\title{
Emergent Yang-Mills theory
}

\author{
Robert de Mello Koch, ${ }^{a, b}$ Jia-Hui Huang, ${ }^{a}$ Minkyoo Kim ${ }^{b}$ and Hendrik J.R. Van Zyl ${ }^{b}$ \\ ${ }^{a}$ Guangdong Provincial Key Laboratory of Nuclear Science, Institute of Quantum Matter, \\ South China Normal University, Higher Education Mega Center, \\ West Waihuan Road No. 378, Guangzhou, China \\ ${ }^{b}$ National Institute for Theoretical Physics, \\ School of Physics and Mandelstam Institute for Theoretical Physics, \\ University of the Witwatersrand, 1 Jan Smuts Avenue, Braamfontein 2000, \\ Johannesburg, South Africa \\ E-mail: robert@neo.phys.wits.ac.za, huangjh@m.scnu.edu.cn, \\ minkyoo.kim@wits.ac.za, hjrvanzyl@gmail.com
}

ABSTRACT: We study the spectrum of anomalous dimensions of operators dual to giant graviton branes. The operators considered belong to the $\operatorname{su}(2 \mid 3)$ sector of $\mathcal{N}=4$ super Yang-Mills theory, have a bare dimension $\sim N$ and are a linear combination of restricted Schur polynomials with $p \sim O(1)$ long rows or columns. In the same way that the operator mixing problem in the planar limit can be mapped to an integrable spin chain, we find that our problems maps to particles hopping on a lattice. The detailed form of the model is in precise agreement with the expected world volume dynamics of $p$ giant graviton branes, which is a $\mathrm{U}(p)$ Yang-Mills theory. The lattice model we find has a number of noteworthy features. It is a lattice model with all-to-all sites interactions and quenched disorder.

Keywords: 1/ $N$ Expansion, AdS-CFT Correspondence

ArXiv ePrint: 2005.02731 


\section{Contents}

1 Introduction 1

2 Operators 3

2.1 Restricted Schur polynomials 3

2.2 Gauss graph basis 6

3 Action of the dilatation operator on restricted Schur polynomials 9

4 Dilatation operator on Gauss graphs $\quad 13$

5 Emergent lattice model $\quad 21$

6 Emergent Yang-Mills theory $\quad 24$

$\begin{array}{lll}7 & \text { Mixing with closed string states } & 27\end{array}$

8 Conclusions and outlook $\quad 30$

A Field redefinition $\quad 32$

B $N^{-1}$ corrections to matrix elements of the dilatation operator 33

\section{Introduction}

The operator mixing problem in the planar limit of $\mathcal{N}=4$ super Yang-Mills theory is solved. This dramatic progress was achieved by mapping the dilatation operator of the theory to the Hamiltonian of an integrable spin chain [1]. The mapping identifies each single trace operator with a state of the spin chain and operators of a definite dimension map to spin chain states with a definite energy. The integrable model describes the dynamics of magnons which can scatter with each other. This scattering between the magnons happens in one dimension. As far as the single trace operators are concerned, reordering fields within the trace corresponds to changing their positions in this single dimension. In the integrable spin chain, this dimension is that of the spin chain lattice, while in the holographically dual theory it is the string world sheet. This is precisely what we should have expected from the AdS/CFT correspondence [2-4]: we know that the planar limit of the gauge theory is dual to perturbative string theory, so we expect the world sheet dynamics of a string to emerge from the planar limit of the CFT.

We expect something similar happens whenever we focus on a class of operators that are holographically dual to a system with a definite semi-classical limit: the dilatation operator should be mapped to the Hamiltonian of the dynamics of the relevant semi-classical physics. 
Our goal in this article is to test this expectation for the class of operators holographically dual to giant graviton branes [5-7]. This class of operators have a bare dimension of order $N[8,9]$. In this regime the single trace operators don't provide a useful starting point for the operator mixing problem. Indeed, for operators with such a large bare dimension mixing between different trace structures is not suppressed [10]. We will start from the basis provided by the restricted Schur polynomials, which is reviewed in section 2 . We study the $\operatorname{su}(2 \mid 3)$ sector of the theory. Truncation to this subsector is consistent to all orders of perturbation theory [11]. This is the maximal closed subsector with finitely many fields. Since there are finitely many fields we are still able to obtain explicit formulas, without too much work. The restricted Schur polynomials that span the $\mathrm{su}(2 \mid 3)$ sector of the theory are labeled by 6 Young diagrams and some multiplicity labels. For operators dual to giant gravitons [5], the Young diagram labels have a small number of long columns and for operators dual to dual giant gravitons $[6,7]$, the Young diagram labels have a small number of long rows [8-10]. These operators diagonalize the free field theory two point function to all orders in $1 / N$ and they mix only weakly at weak coupling. In section 3 we derive an exact formula for the action of the one loop dilatation operator on restricted Schur polynomials that span the $\mathrm{su}(2 \mid 3)$ sector of the theory. This is the first new result in this paper. The novel ingredients involve the mixing of fermions, which was not considered in previous studies. We find a rather simple way to express the complete result. This result is exact in $1 / N$. By specializing to the operators dual to system of giant gravitons, in section 4 we use simplifications of large $N$. These simplifications suggest a new basis labeled by two Young diagrams and a graph, the so called Gauss graph operators [12]. The nodes of the graph correspond to the rows/columns of the Young diagram label. There are also edges stretched between nodes in the graph and edges that have both end points on a given node. We have derived a formula for the action of the dilatation operators in the Gauss graph basis of the $\mathrm{su}(2 \mid 3)$ sector. This is the second new result in this paper. Matrix elements of the dilatation operator are given in terms of the number of edges between specific nodes on the graph. Further, the dilatation operators preserves the number of edges stretched between nodes but can change the number of edges with both endpoints attached to a given node.

This dilatation operator is rewritten in section 5 as a lattice model for particles. The basic idea is simply to introduce oscillator creation and annihilation operators and then to rewrite the number of edges in terms of these oscillators. We demonstrate in section 6 that the resulting Hamiltonian is in detailed agreement with the Yang-Mills theory expected as the world volume dynamics of the giant graviton branes. Each Gauss graph operator becomes a state in a Fock space, with the graph giving an occupation number representation of the states of the emergent world volume gauge theory. This is the central result in this paper and it proves that the dilatation operator is mapped to the Hamiltonian of the dynamics of the semi-classical physics of giant graviton branes. Section 8 contains some conclusions and a discussion of our results, which make a number of concrete suggestions. For example, to explore the thermodynamics of the gravity theory dual to these large dimension operators, we argue that one is considering the dynamics of a lattice model with all-to-all sites interactions and quenched disorder. This looks a lot like the dynamics of 
the SYK model and our study may shed some light on the holographic relevance of models with quenched disorder.

\section{Operators}

We use two bases of operators in this study. A formula for matrix elements of the dilatation operator in the restricted Schur polynomial basis is the starting point for our study. The formula we obtain is exact, meaning that it does not use any of the simplifications of large $N$. Specializing to operators with bare dimension of order $N$, labeled by Young diagrams with order 1 long rows or columns, naturally leads to a second basis for this class of operators, known as the Gauss graph operators. Working in this basis allows us to exploit simplifications of large $N$. Both bases are introduced and explained in this section.

\subsection{Restricted Schur polynomials}

Restricted Schur polynomials [13-15], have their genesis in permutations groups and their representations, as well as in combinatorics of gauge invariant operators in multi-matrix models. Although we will not use them in our study, note that closely related bases were introduced and studied in [16-19]. Restricted Schur polynomials are labeled by a collection of Young diagrams and multiplicity labels, as we explain below. They provide a basis for local gauge invariant operators of the theory, account for finite $N$ relations and diagonalize (to all orders in $1 / N$ ) the free field theory two point function $[15,20]$. When interactions are turned on, they only mix very weakly: at $L$-loops two operators will only mix if their labels differ by moving at most $L$ boxes in any of the Young diagrams in the label [21, 22]. ${ }^{1}$ It is for these reasons that restricted Schur polynomials provide an attractive basis within which the operator mixing problem can be formulated.

We truncate to the $\operatorname{su}(2 \mid 3)$ sector of $\mathcal{N}=4$ super Yang-Mills theory. Consequently, the Schur polynomials we study are constructed using three adjoint boson fields and two adjoint fermion fields. Denote the bosonic fields by $\phi_{i}$ with $i=1,2,3$ and the fermionic fields by $\psi_{a}$ with $a=1,2$. The complete set of gauge invariant observables is obtained by taking arbitrary products of these fields and then, to produce a gauge invariant, contracting row and column indices of the fields, in all possible ways. Permuting row indices before tracing, we obtain all possible gauge invariant operators, with all possible trace structures. We can label operators with the permutation that was performed on the row indices. This labeling is redundant as a consequence of symmetries in the problem: swapping identical fields does not lead to distinct observables. Thus, there are two permutations groups that naturally enter the problem: the permutation group swapping row indices before tracing and the permutation group swapping identical fields. Consider operators constructed using $n_{i}$ of the $\phi_{i}$ fields and $m_{a}$ of the $\psi_{a}$ fields. In what follows we will use the indices $A, \hat{A}$ running over the fields $A=\left\{\phi_{1}, \phi_{2}, \phi_{3}, \psi_{1}, \psi_{2}\right\}$ and $\hat{A}=\left\{\phi_{2}, \phi_{3}, \psi_{1}, \psi_{2}\right\}$. The permutation group swapping identical fields is given by the following product of symmetric groups

$$
\mathcal{G}_{\mathrm{symm}}=S_{n_{1}} \times S_{n_{2}} \times S_{n_{3}} \times S_{m_{1}} \times S_{m_{2}}
$$

\footnotetext{
${ }^{1}$ See also $[23,24]$.
} 
The second permutation group that plays a role is the symmetric group $S_{n_{T}}$ which swaps row indices before tracing. Here $n_{T}=n_{1}+n_{2}+n_{3}+m_{1}+m_{2}$ is the total number of fields appearing in the operator. We must take the symmetry $\mathcal{G}_{\text {symm }}$ into account to obtain a non-redundant labeling of the gauge invariant operators. This is done by recognizing that permutations labeling distinct observables belong to distinct restricted conjugacy classes, as we now explain. First, we will define the notion of a restricted conjugacy class: given a group $G$ and subgroup $H, g_{1}, g_{2} \in G$ are in the same restricted conjugacy class if and only if $g_{1}=h g_{2} h^{-1}$ for some $h \in H$ [14]. The operator corresponding to a permutation $\sigma$ is given by

$$
\begin{aligned}
& \operatorname{Tr}\left(\sigma \psi_{1}^{\otimes m_{1}} \psi_{2}^{\otimes m_{2}} \phi_{3}^{\otimes n_{3}} \phi_{2}^{\otimes n_{2}} \phi_{1}^{\otimes n_{1}}\right)=\psi_{1 i_{\sigma(1)}}^{i_{1}} \cdots \psi_{1 i_{\sigma\left(m_{1}\right)}}^{i_{m_{1}}} \psi_{2 i_{\sigma\left(m_{1}+1\right)}}^{i_{m_{1}+1}} \cdots \psi_{2 i_{\sigma\left(m_{1}+m_{2}\right)}}^{i_{m_{1}+m_{2}}} \\
& \times \phi_{3} i_{i_{\sigma\left(m_{1}+m_{2}+1\right)}}^{i_{m_{1}+m_{2}+1}} \cdots \phi_{3 i_{\sigma\left(m_{1}+m_{2}+n_{3}\right)}}^{i_{m_{1}+m_{2}+n_{3}}} \phi_{2_{i_{\sigma\left(m_{1}+m_{2}+n_{3}+1\right)}}^{i_{m_{1}+m_{2}+n_{3}+1}}}^{i_{m_{1}}} \cdots \phi_{i_{\sigma\left(m_{1}+m_{2}+n_{3}+n_{2}\right)}}^{i_{m_{1}+m_{2}+n_{3}}+n_{2}}
\end{aligned}
$$

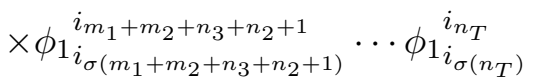

The $\psi_{1}$ fields are the first $m_{1}$ factors in the tensor product and the $\psi_{2}$ fields the next $m_{2}$ factors and so on. We say that the $\psi_{2}$ fields, for example, occupy slots $m_{1}+1$ to $m_{1}+m_{2}$. We will now argue that if we choose $G=S_{n_{T}}$ and $H=\mathcal{G}_{\text {symm }}$, then the difference between two permutations in a given restricted conjugacy class is a permutation swapping identical fields so that they do indeed give identical (possibly up to a sign for fermions) gauge invariant operators. The result follows immediately after using the easily verified identity

$$
\operatorname{Tr}\left(\rho^{-1} \sigma \rho A_{1} A_{2} \cdots A_{n_{T}}\right)=\operatorname{Tr}\left(\sigma A_{\rho(1)} A_{\rho(2)} \cdots A_{\rho\left(n_{T}\right)}\right)
$$

This does not quite remove the complete set of redundancies: observables that naively look independent can be linearly dependent at finite $N$. As an example, the Cayley-Hamilton theorem tells us that any square matrix over a commutative ring satisfies its own characteristic equation. Taking a trace of this equation gives an identity between different multi trace structures, proving they are not linearly independent. To take these finite $N$ relations into account, perform a Fourier transform on the space of restricted conjugacy classes. In the end, each field is in an irreducible representation of the permutation group permuting that species of field, and in an irreducible representation of the permutation group permuting the entire collection of fields. Since irreducible representations of permutation groups are labeled by Young diagrams there is one Young diagram label for each species of field and one additional Young diagram for the complete collection of fields. In addition, there are multiplicity labels. These multiplicity labels are needed because upon restricting an irreducible representation of the group permuting the complete set of fields in the operator, to the group that permutes only identical fields, many copies of a given irreducible representation of the subgroup might arise. Finite $N$ relations force polynomials labeled by a Young diagram with more than $N$ rows to vanish. Thus, by keeping the restricted Schur polynomials, labeled by Young diagrams with at most $N$ rows, we obtain a basis for the local gauge invariant operators.

For the $\mathrm{su}(2 \mid 3)$ sector, the restricted Schur polynomials are given by [25]

$$
\chi_{R,(\vec{r}, \vec{s}) \vec{\alpha} \vec{\beta}}\left(\phi_{i}, \psi_{a}\right)=\frac{1}{n_{1} ! n_{2} ! n_{3} ! m_{1} ! m_{2} !} \sum_{\sigma \in S_{n_{T}}} \chi_{R,(\vec{r}, \vec{s}) \vec{\alpha} \vec{\beta}}(\sigma) \operatorname{Tr}\left(\sigma \psi_{1}^{\otimes m_{1}} \psi_{2}^{\otimes m_{2}} \phi_{3}^{\otimes n_{3}} \phi_{2}^{\otimes n_{2}} \phi_{1}^{\otimes n_{1}}\right)
$$


Each operator is indexed by a collection $R,(\vec{r}, \vec{s}) \vec{\alpha} \vec{\beta}$ of labels. We know that swapping identical bosons is a symmetry, so we want a simultaneous swap of row and column indices of bosons in the operator to leave the operator invariant. The only way to get the invariant is to place row and column indices into the same representation $r$ and then project to the (unique) invariant in $r \times r$. Thus, the row and column indices of each bosonic field are in a definite representation. The indices of the $\phi_{i}$ fields are in representation ${ }^{2} r_{i} \vdash$ $n_{i}$. For the fermionic fields we need to place the fermions into a totally antisymmetric representation, and this is achieved by placing the row indices into some representation $s$ and the column indices into the conjugate representation $s^{T}$ and then projecting to the (unique) antisymmetric representation appearing in $s \times s^{T}$ as explained in [25]. The conjugate representation is obtained by flipping the Young diagram so that row and column lengths are exchanged. We place the row indices of the $\psi_{a}$ 's into representation $s_{a} \vdash m_{1}$ and the column indices into $s_{a}^{T}$. The complete set of fields are in representation $R \vdash n_{T}$. To refer to collections of Young diagrams we will use the notation $\vec{r}$ and $\vec{s}$, etc. The collection $(\vec{r}, \vec{s})$ specifies a representation of $\mathcal{G}_{\text {symm }}$ which is a subgroup of $S_{n_{T}}$. The representation of the subgroup is a subspace of the carrier space of representation $R$. At this point we are forced to introduce multiplicities because the representation of the subgroup may appear more than once. We imagine embedding the subspace by removing $m_{1}$ boxes ${ }^{3}$ from $R$, and assembling them into representation $s_{1}$ and $s_{1}^{T}$. There may be more than one way to do this, so that there may be more than one copy of these spaces. Distinguish the different copies using the labels $\alpha_{3}$ (for $s_{1}$ ) and $\beta_{3}$ (for $s_{1}^{T}$ ). Next $m_{2}$ boxes are removed and assembled into $s_{2}$ and $s_{2}^{T}$, with multiplicities $\alpha_{4}$ and $\beta_{4}$. The next $n_{3}$ boxes are removed and assembled into $r_{3}$ with multiplicities $\alpha_{2}$ and $\beta_{2}$ and finally, $n_{2}$ boxes are removed and assembled into $r_{2}$ with multiplicities $\alpha_{1}$ and $\beta_{1}$. The last $n_{1}$ boxes remaining in $R$ are identified with $r_{1}$ so that $r_{1}$ is multiplicity free.

$\chi_{R,(\vec{r}, \vec{s}) \vec{\alpha} \vec{\beta}}(\sigma)$ is a restricted character [23], obtained by summing the row index of $\Gamma^{R}(\sigma)$ over the subspace $(\vec{r}, \vec{s}) \vec{\alpha}$ and the column index over the subspace $(\vec{r}, \vec{s}) \vec{\beta}$ which both arise upon restricting irreducible representation $R$ of $S_{n_{T}}$ to its $S_{n_{1}} \times S_{n_{2}} \times S_{n_{3}} \times S_{m_{1}} \times S_{m_{2}}$ subgroup, as we have just explained in detail. The reader can consult [15] for further details and results. Here we simply note that the restricted characters are a complete set of functions on the restricted conjugacy classes, so that the formula (2.4) can be understood as a Fourier transform from the space of restricted conjugacy classes, to the space of Young diagrams and multiplicity labels. This interpretation relies on basic ideas first introduced in the pioneering paper [17]. Even at finite $N$, restricted Schur polynomials are linearly independent $[15,20]$ and diagonalize the free field theory two point function [15]. A straight forward computation now shows that

$$
\left\langle\chi_{R,(\vec{r}, \vec{s}) \vec{\alpha} \vec{\beta}}\left(\phi_{i}, \psi_{a}\right) \chi_{T,(\vec{t}, \vec{u}) \vec{\gamma} \vec{\delta}}^{\dagger}\left(\phi_{i}, \psi_{a}\right)\right\rangle=\delta_{R T} \delta_{\vec{r} \vec{t}} \delta_{\vec{s} \vec{u}} \delta_{\vec{\alpha} \vec{\gamma}} \delta_{\vec{\beta} \vec{\delta}} \frac{f_{R} \operatorname{hooks}_{R}}{\prod_{m} \text { hooks }_{r_{m}} \prod_{n} \text { hooks }_{s_{n}}}
$$

\footnotetext{
${ }^{2}$ The notation $r \vdash n$ means that $r$ is a partition of $n$. Every Young diagram can be understood as a partition of an integer with the parts recording how many boxes there are in each row of the diagram. Consequently we write $r \vdash n$ to state that $r$ is a Young diagram with $n$ boxes.

${ }^{3}$ Each box corresponds to a field. Thus, each box corresponds to a row index and a column index and the collection of row and column indices must each be put into an irreducible representation. This is why in the discussion that follows we assemble the boxes into two representations, each with their own multiplicity label.
} 
where we are using the obvious notation

$$
\delta_{\vec{r} \vec{t}}=\prod_{i=1}^{3} \delta_{r_{i} t_{i}} \quad \delta_{\vec{s} \vec{u}}=\prod_{a=1}^{2} \delta_{s_{a} u_{a}} \quad \delta_{\vec{\alpha} \vec{\gamma}} \delta_{\vec{\beta} \vec{\delta}}=\prod_{k=1}^{4} \delta_{\alpha_{k} \gamma_{k}} \delta_{\beta_{k} \delta_{k}}
$$

Simple counting arguments prove that the number of restricted Schur polynomials matches the number of gauge invariant operators that can be defined [25].

In working with the restricted character it is useful to write

$$
\chi_{R,(\vec{r}, \vec{s}) \vec{\alpha} \vec{\beta}}(\sigma)=\operatorname{Tr}_{R}\left(P_{R,(\vec{r}, \vec{s}) \vec{\alpha} \vec{\beta}} \Gamma^{(R)}(\sigma)\right)
$$

The trace is over the carrier space of irreducible representation $R$. The operator $P_{R,(\vec{r}, \vec{s}) \vec{\alpha} \vec{\beta}}$ is an intertwining map, which sends the row indices of $\Gamma^{(R)}(\sigma)$ to the $\vec{\beta}$ copy of $\left(\vec{r}, \vec{s}^{T}\right)$ and the column indices to the $\vec{\alpha}$ copy of $(\vec{r}, \vec{s})$ in the above trace. The intertwining maps obey

$$
\begin{gathered}
\operatorname{sgn}\left(\rho_{1}\right) \operatorname{sgn}\left(\rho_{2}\right) P_{R,(\vec{r}, \vec{s}) \vec{\alpha} \vec{\beta}} \Gamma_{r_{1}}\left(\sigma_{1}\right) \otimes \Gamma_{r_{2}}\left(\sigma_{2}\right) \otimes \Gamma_{r_{3}}\left(\sigma_{3}\right) \otimes \Gamma_{s_{1}^{T}}\left(\rho_{1}\right) \otimes \Gamma_{s_{2}^{T}}\left(\rho_{2}\right) \\
=\Gamma_{r_{1}}\left(\sigma_{1}\right) \otimes \Gamma_{r_{2}}\left(\sigma_{2}\right) \otimes \Gamma_{r_{3}}\left(\sigma_{3}\right) \otimes \Gamma_{s_{1}}\left(\rho_{1}\right) \otimes \Gamma_{s_{2}}\left(\rho_{2}\right) P_{R,(\vec{r}, \vec{s}) \vec{\alpha} \vec{\beta}}
\end{gathered}
$$

as well as

$$
P_{R_{1},\left(\vec{r}_{1}, \vec{s}_{1}\right) \vec{\alpha}_{1} \vec{\beta}_{1}} P_{R_{2},\left(\vec{r}_{2}, \vec{s}_{2}\right) \vec{\alpha}_{2} \vec{\beta}_{2}}^{\dagger}=\delta_{R_{1} R_{2}} \delta_{\vec{r}_{1} \vec{r}_{2}} \delta_{\vec{s}_{1} \vec{s}_{2}} \delta_{\vec{\alpha}_{2} \vec{\beta}_{1}} \bar{P}_{R_{1},\left(\vec{r}_{1}, \vec{s}_{1}\right) \vec{\alpha}_{1} \vec{\beta}_{2}}
$$

and they can be written as a tensor product as follows

$$
\bar{P}_{R,(\vec{r}, \vec{s}) \vec{\alpha} \vec{\beta}}=p_{r_{1}} \otimes p_{r_{2} \alpha_{1} \beta_{1}} \otimes p_{r_{3} \alpha_{2} \beta_{2}} \otimes p_{s_{1} \alpha_{3} \beta_{3}} \otimes p_{s_{2} \alpha_{4} \beta_{4}}
$$

Finally, we find it convenient to work with restricted Schur polynomials normalized to have a unit two point function. The normalized operators are defined by

$$
\chi_{R,(\vec{r}, \vec{s}) \vec{\alpha} \vec{\beta}}\left(\phi_{i}, \psi_{a}\right)=\sqrt{\frac{f_{R} \operatorname{hooks}_{R}}{\prod_{m} \operatorname{hooks}_{r_{m}} \prod_{n} \operatorname{hooks}_{s_{n}}}} O_{R,(\vec{r}, \vec{s}) \vec{\alpha} \vec{\beta}}\left(\phi_{i}, \psi_{a}\right)
$$

\subsection{Gauss graph basis}

We now specialize to operators which have a definite semi-classical limit in the holographically dual theory. ${ }^{4}$ Doing so will allow us to exploit the simplifications of large $N$ and, for this class of operators, a diagonalization of the one loop dilatation operator. The operators we consider have a dimension $\Delta \sim N$ so that the Young diagram $R$ labeling the operator has $\sim N$ boxes. In addition $R$ has a fixed $\sim 1$ number of rows or columns. Operators with $p$ long columns are dual to a system of $p$ giant gravitons and operators with $p$ long rows are dual to $p$ dual giant gravitons. ${ }^{5}$ These operators mix with each other, but not with operators labeled by Young diagrams that have a different number of rows or columns. In what follows, for simplicity we will discuss the case of long rows. There is an identical discussion for long columns. We consider operators constructed using mainly $\phi_{1}$ fields, so that

\footnotetext{
${ }^{4}$ Specializing to classes of operators is always necessary. Even in the planar limit one is forced to restrict attention to operators of dimension $\Delta$ with $\Delta^{2} / N \ll 1$.

${ }^{5}$ Branes connected by an open string described using a spin chain have been considered in [26-30].
} 
$n_{1} \sim N$. In addition, there are some bosonic $\phi_{2}, \phi_{3}$ excitations, as well fermionic $\psi_{1}, \psi_{2}$ excitations. The number of excitations is limited by fixing $n_{2} \sim n_{3} \sim m_{1} \sim m_{2} \sim \sqrt{N}$.

A key observation motivating the Gauss graph basis concerns the shape of the $R$ Young diagram of the generic operator: almost all operators in this class have unequal row lengths. The difference in the length of any two distinct rows in $R$ is generically of size $a N$, where $a$ is a number of order 1 , possibly with $a \ll 1$. The one loop dilatation operator moves a single box at a time so that of the order of $N$ applications are required to produce operators with equal row lengths. Thus, at weak coupling, if we start with sufficiently unequal lengths, we always have unequal lengths. The conclusion is that, at large $N$ and weak coupling, corners on the right hand side of the Young diagram are well separated. This limit was introduced and studied in $[22,31]$ where it was called the displaced corners limit. The action of the symmetric group on right most boxes simplifies in this limit: after neglecting order $1 / N$ corrections, permutations simply swap boxes they act on $[22,32]$. These are precisely the boxes that are to be removed and reassembled into irreducible representations of the subgroup which is why this simplification has far reaching consequences. The simplified action implies both new symmetries and new conservation laws. Swapping row or column indices of fields of a given species, that belong to the same row, is a new symmetry. The new conservation law manifests as the fact that operators only mix if they have the same number of excitation fields of each species in a given row. This new conservation law implies that we can refine the number of fields of a given species $N_{\hat{A}}$ to produce a $p$ dimensional vector $\vec{N}_{\hat{A}}$, with each component recording how many fields are in a given row. For example, the number of $\phi_{2}$ fields $n_{2}$ is refined to produce the vector $\vec{n}_{2}$. The vector $\vec{n}_{2}$ labeling the dilatation operator is preserved so that an operator with vector $\vec{n}_{2}$ will not mix with a second operator with $\vec{n}_{2}^{\prime}$ if $\vec{n}_{2} \neq \vec{n}_{2}^{\prime}$. The group swapping $\phi_{2}$ fields in a given row, which is the enhanced symmetry of the displaced corners limit, is

$$
H_{\vec{n}_{2}}=S_{\left(n_{2}\right)_{1}} \times S_{\left(n_{2}\right)_{2}} \times \cdots \times S_{\left(n_{2}\right)_{p}}
$$

This symmetry acts independently on the row and column indices, so that the $\phi_{2}$ fields can be parametrized by a permutation belonging to the double coset

$$
H_{\vec{n}_{2}} \backslash S_{n_{2}} / H_{\vec{n}_{2}}
$$

The number of values that the triple $\left(r_{2}, \alpha_{1}, \beta_{1}\right)$ takes equals the order of the double coset $H_{\vec{n}_{2}} \backslash S_{n_{2}} / H_{\vec{n}_{2}}$, suggesting that instead of organizing the $\phi_{2}$ fields with the $r_{2}, \alpha_{1}, \beta_{1}$ labels, we can organize them using the elements of the double coset [12]. This is indeed the case, and the resulting basis is the Gauss graph basis. The double cosets that are relevant for labeling our operators are given by

$$
\begin{aligned}
& \phi_{2} \leftrightarrow \sigma_{\phi_{2}} \in H_{\vec{n}_{2}} \backslash S_{n_{2}} / H_{\vec{n}_{2}} \\
& \phi_{3} \leftrightarrow \sigma_{\phi_{3}} \in H_{\vec{n}_{3}} \backslash S_{n_{3}} / H_{\vec{n}_{3}} \\
& \psi_{1} \leftrightarrow \sigma_{\psi_{1}} \in H_{\vec{m}_{1}} \backslash S_{m_{1}} / H_{\vec{m}_{1}} \\
& \psi_{2} \leftrightarrow \sigma_{\psi_{2}} \in H_{\vec{m}_{2}} \backslash S_{m_{2}} / H_{\vec{m}_{2}}
\end{aligned}
$$


When we want to refer to a collection of permutations, one from each of the double cosets above, we will use the notation $\vec{\sigma}=\left(\sigma_{\phi_{2}}, \sigma_{\phi_{3}}, \sigma_{\psi_{1}}, \sigma_{\psi_{2}}\right)$.

Gauss graph operators [12] are labeled by two Young diagrams (the $R$ and $r_{1}$ labels of the restricted Schur polynomial) and a graph. Nodes of the graph correspond to rows/columns of Young diagram $r_{1}$. Each $\hat{A}$ field type $\left(\phi_{2}, \phi_{3}, \psi_{1}\right.$ or $\left.\psi_{2}\right)$ corresponds to a species of edge in the graph and there is an edge for each field. The edges are directed and stretch between nodes. An edge is allowed to leave and then return to the same node. It is both convenient and possible to decompose the complete graph, to give a graph for each $\hat{A}$. We can label the graphs using permutations, but this labeling is again redundant due to symmetries. Swapping edges that terminate on a given node, or emanate from a given node is a symmetry. This observation can be exploited to show that graphs are enumerated by elements of a double coset. We refer the reader to [33] for the details. So the appearance of double cosets in the displaced corners limit naturally leads to the graph labeling the operator. The complete collection of graphs with $n$ edges and $p$ nodes, and with number of edges terminating at each node recorded in $\vec{n}$ is described by a particular double coset. The elements of the double cosets recorded in (2.14) correspond to the graphs we consider [33]. We argue below that the number of edges give an occupation number description of the fields of the emergent gauge theory defined on the world volume of the giant gravitons. Consequently they must reflect constraints implied by the Gauss Law [13, 34] which manifests as the fact that only graphs with the same number of edges terminating on a node as number of edges emanating from a node, for each species, are allowed. This is the origin of the name Gauss graph [12]. Fermi statistics forbids two or more parallel edges (i.e. edges with the same orientation and endpoints) of the same fermion species [35]. We refined $N_{\hat{A}}$ to produce a vector $\vec{N}_{\hat{A}}$. To describe the graph we refine $\vec{N}_{\hat{A}}$ further to produce a matrix $\left(N_{\hat{A}}\right)_{i \rightarrow j}$ whose elements describe the number of edges running from node $i$ to node $j$. We will abbreviate $\left(N_{\hat{A}}\right)_{i \rightarrow i}$ as $\left(N_{\hat{A}}\right)_{i i}$. The total number of edges between nodes $i$ and $j$ is given by $\left(N_{\hat{A}}\right)_{i j}=\left(N_{\hat{A}}\right)_{i \rightarrow j}+\left(N_{\hat{A}}\right)_{j \rightarrow i}$.

The orthogonal transformation from the restricted Schur polynomial basis to the Gauss graph basis uses two types of group theoretic coefficients. The first set of coefficients

$$
C_{\mu_{1} \mu_{2}}^{(r)}(\tau)=\left|H_{\vec{n}}\right| \sqrt{\frac{d_{r}}{n !}} \sum_{k, m=1}^{d_{r}} \Gamma^{(r)}(\tau)_{k m} B_{k \mu_{1}}^{r \rightarrow 1_{H_{\vec{n}}}} B_{m \mu_{2}}^{r \rightarrow 1_{H_{\vec{n}}}}
$$

are used to transform the labels of the $\phi_{2}, \phi_{3}$ fields. In this formula $d_{r}$ is the dimension of irreducible representation $r \vdash n$ of $S_{n}, \Gamma^{(r)}(\tau)_{k m}$ is the matrix representing $\tau \in S_{n}$ in irreducible representation $r$ and

$$
\left|H_{\vec{n}}\right|=n_{1} ! n_{2} ! \cdots n_{p} !
$$

is the order of the group $H_{\vec{n}}$. Finally, $B_{k \mu_{1}}^{r \rightarrow 1_{H_{\vec{n}}}}$ is a branching coefficient, described in more detail below. The second set of group theoretic coefficients, distinguished by a tilde,

$$
\tilde{C}_{\mu_{1} \mu_{2}}^{(s)}(\tau)=\left|H_{\vec{m}}\right| \sqrt{\frac{d_{s}}{m !}} \sum_{k, m=1}^{d_{s}}\left(\Gamma^{(s)}(\tau) \hat{O}\right)_{k m} B_{k \mu_{1}}^{s \rightarrow 1_{H_{\vec{m}}}} B_{m \mu_{2}}^{s^{T} \rightarrow 1_{H_{\vec{m}}}^{m}}
$$


are used to transform the $\psi_{1}, \psi_{2}$ labels. We have introduced another set of branching coefficients, which are also discussed in more detail below, as well as an operator $\hat{O}$, which maps from irreducible representation $s^{T}$ to $s$ and is normalized so that (here 1 is the identity permutation)

$$
\hat{O}^{T} \hat{O}=\Gamma^{\left(s^{T}\right)}(1)
$$

In terms of these coefficients, the Gauss graph operators are

$$
O_{R, r_{1}}(\vec{\sigma})=\sum_{r_{2} \vdash n_{2}} \sum_{r_{3} \vdash n_{3}} \sum_{s_{1} \vdash m_{1}} \sum_{s_{2} \vdash m_{2}} \sum_{\vec{\mu}, \vec{\nu}} C_{\mu_{1} \nu_{1}}^{\left(r_{2}\right)}\left(\sigma_{\phi_{2}}\right) C_{\mu_{2} \nu_{2}}^{\left(r_{3}\right)}\left(\sigma_{\phi_{3}}\right) \tilde{C}_{\mu_{3} \nu_{3}}^{\left(s_{1}\right)}\left(\sigma_{\psi_{1}}\right) \tilde{C}_{\mu_{4} \nu_{4}}^{\left(s_{2}\right)}\left(\sigma_{\psi_{2}}\right) O_{R,(\vec{r}, \vec{s}) \vec{\mu} \vec{\nu}}
$$

In performing the basis change, the basic formulas that we need are properties of the branching coefficients which we will now review. The branching coefficients introduced above are defined by

$$
\begin{aligned}
\sum_{\mu} B_{k \mu}^{s \rightarrow 1_{H}} B_{l \mu}^{s \rightarrow 1_{H}} & =\frac{1}{|H|} \sum_{\gamma \in H} \Gamma^{(s)}(\gamma)_{k l} \\
\sum_{\mu} B_{k \mu}^{s^{T} \rightarrow 1^{m}} B_{l \mu}^{s^{T} \rightarrow 1^{m}} & =\frac{1}{|H|} \sum_{\gamma \in H} \operatorname{sgn}(\gamma) \Gamma^{\left(s^{T}\right)}(\gamma)_{k l}
\end{aligned}
$$

The coefficients $B_{l \mu}^{s \rightarrow 1_{H}}$ resolve the multiplicities that arise when we restrict irreducible representation $s$ of $S_{m}$ to the identity representation $1_{H}$ of $H$ for which $\Gamma^{1_{H}}(\gamma)=1 \forall \gamma$. The coefficients $B_{l \mu}^{s \rightarrow 1^{m}}$ resolve the multiplicities that arise when we restrict irreducible representation $s$ of $S_{m}$ to representation $1^{m}$ of $H$ for which $\Gamma^{1^{m}}(\gamma)=\operatorname{sgn}(\gamma) \forall \gamma$. These branching coefficients are not independent: $B_{n \mu}^{s \rightarrow 1_{H}} O_{n l}=B_{l \mu}^{s^{T} \rightarrow 1^{m}}$. This relation between the two implies that the transformation to Gauss graph basis is exactly the same for the fermions and bosons [35]

$$
\tilde{C}_{\mu_{1} \mu_{2}}^{\left(s_{i}\right)}(\tau)=C_{\mu_{1} \mu_{2}}^{\left(s_{i}\right)}(\tau)
$$

When evaluating matrix elements of the dilatation operator in the Gauss graph basis, it is convenient to work with operators $\hat{O}_{R, r}(\vec{\sigma})$ normalized to have a unit two point function. They are related to the operators we have just defined as follows

$$
O_{R, r}(\vec{\sigma})=\sqrt{\prod_{\hat{A}=1}^{4} \prod_{i, j=1}^{p}\left(N_{\hat{A}}\right)_{i \rightarrow j} !} \hat{O}_{R, r}(\vec{\sigma})
$$

\section{Action of the dilatation operator on restricted Schur polynomials}

The one loop dilatation operator in the $\mathrm{su}(2 \mid 3)$ sector is given by $[36,37]$

$$
\begin{aligned}
D= & -\frac{2 g_{\mathrm{YM}}^{2}}{(4 \pi)^{2}}\left(\sum_{i>j=1}^{3} \operatorname{Tr}\left(\left[\phi_{i}, \phi_{j}\right]\left[\partial_{\phi_{i}}, \partial_{\phi_{j}}\right]\right)+\sum_{i=1}^{3} \sum_{a=1}^{2} \operatorname{Tr}\left(\left[\phi_{i}, \psi_{a}\right]\left[\partial_{\phi_{i}}, \partial_{\psi_{a}}\right]\right)\right. \\
& \left.+\operatorname{Tr}\left(\left\{\psi_{1}, \psi_{2}\right\}\left\{\partial_{\psi_{1}}, \partial_{\psi_{2}}\right\}\right)\right)
\end{aligned}
$$


It is useful to introduce the notation

$$
D \equiv-\frac{2 g_{\mathrm{YM}}^{2}}{(4 \pi)^{2}} \sum_{A>B=1}^{5} D_{A B}
$$

where $D_{A B}$ mixes fields of species $A$ and $B$. To derive the action of $D$ on the restricted Schur polynomials, we need to evaluate the derivatives and then express the result as a linear combination of restricted Schur polynomials. The second step is always possible because the restricted Schur polynomials provide a basis. In practice it is carried out using properties of restricted characters that imply [20]

$\operatorname{Tr}\left(\sigma \psi_{1}^{\otimes m_{1}} \psi_{2}^{\otimes m_{2}} \phi_{1}^{\otimes n_{1}} \phi_{2}^{\otimes n_{2}} \phi_{3}^{\otimes n_{3}}\right)=\sum_{R,(\vec{r}, \vec{s}) \vec{\alpha} \vec{\beta}} \frac{d_{R} n_{1} ! n_{2} ! n_{3} ! m_{1} ! m_{2} !}{d_{r_{1}} d_{r_{2}} d_{r_{3}} d_{s_{1}} d_{s_{2}} n_{T} !} \chi_{R,(\vec{r}, \vec{s}) \vec{\alpha} \vec{\beta}}\left(\sigma^{-1}\right) \chi_{R,(\vec{r}, \vec{s}) \vec{\beta} \vec{\alpha}}\left(\phi_{i}, \psi_{a}\right)$

Matrix elements arising from the mixing of two bosonic fields have been derived in [21], while matrix elements for the mixing of a boson and fermion field were derived in [25]. Matrix elements relevant for the mixing of two fermionic fields have not been considered previously so we will discuss their derivation in detail below. To simplify the notation, introduce the following shorthand

$$
\begin{array}{llrl}
1_{\psi_{1}} & =1 & m_{\psi_{1}} & =m_{1} \\
1_{\psi_{2}} & =m_{1}+1 & m_{\psi_{2}} & =m_{1}+m_{2} \\
1_{\phi_{3}} & =m_{1}+m_{2}+1 & n_{\phi_{3}} & =m_{1}+m_{2}+n_{3} \\
1_{\phi_{2}} & =m_{1}+m_{2}+n_{3}+1 & n_{\phi_{2}} & =m_{1}+m_{2}+n_{3}+n_{2} \\
1_{\phi_{1}} & =m_{1}+m_{2}+n_{3}+n_{2}+1 & n_{\phi_{1}} & =m_{1}+m_{2}+n_{3}+n_{2}+n_{1}=n_{T}
\end{array}
$$

Due to the presence of fermionic fields we need to be careful about signs. To evaluate the derivatives we need to compute

$$
\begin{aligned}
& A=\left\{\psi_{1}, \psi_{2}\right\}_{j}^{i}\left(\frac{d}{d \psi_{1}^{k}} \frac{d}{d \psi_{2 k}^{i}}+\frac{d}{d \psi_{2}^{k}} \frac{d}{d \psi_{1}^{i}}\right) \sum_{\sigma \in S_{n_{T}}} \operatorname{Tr}_{(\vec{r}, \vec{s}) \vec{\mu} \vec{\nu}}\left(\Gamma^{(R)}(\sigma)\right) \psi_{1 i_{\sigma\left(1_{\psi_{1}}\right)}}^{i_{1_{\psi_{1}}}} \cdots \psi_{1 i_{\sigma\left(m_{\psi_{1}}\right)}}^{i_{m_{\psi_{1}}}}
\end{aligned}
$$

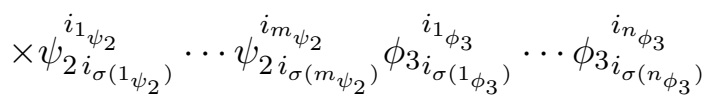

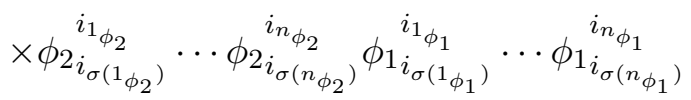

$$
\begin{aligned}
& =m_{1} m_{2} \sum_{\sigma \in S_{n_{T}}} \operatorname{Tr}_{(\vec{r}, \vec{s}) \vec{\mu} \vec{\nu}}\left(\Gamma^{(R)}\left(\left[\left(1_{\psi_{2}}, 1\right), \sigma\right]\right)\right)(-1)^{m_{1}}\left\{\psi_{1}, \psi_{2}\right\}_{i_{\sigma\left(\psi_{\psi_{1}}\right)}}^{i_{1} \psi_{1}} \psi_{\left.1 i_{\sigma\left(1+\psi_{\psi_{1}}\right.}\right)}^{i_{1+1} \psi_{1}} \cdots \psi_{1 i_{\sigma\left(m_{\psi_{1}}\right)}}^{i_{m_{\psi_{1}}}}
\end{aligned}
$$

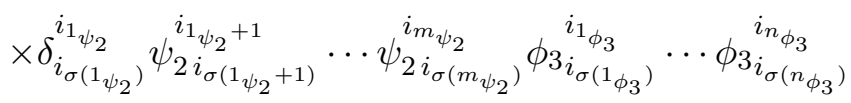

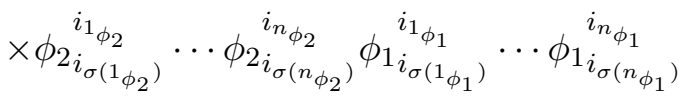

It will prove to be useful to have both indices of the Kronecker delta in the first slot, as this will allow us to express the sum of $S_{n_{T}}$ as a sum over the subgroup $S_{n_{T}-1}$ and its 
cosets. To achieve this, change summation variables from $\sigma$ to $\rho$ where $\sigma=\left(1,1_{2}\right) \rho\left(1,1_{2}\right)$ and then relabel the summation variable back to the original name $\sigma$. The result is

$$
\begin{aligned}
& A=m_{1} m_{2} \sum_{\sigma \in S_{n_{T}}} \operatorname{Tr}_{(\vec{r}, \vec{s}) \vec{\mu} \vec{\nu}}\left(\Gamma^{(R)}\left(\left[\sigma,\left(1,1_{\psi_{2}}\right)\right]\right)\right) \delta_{i_{\sigma(1)}}^{i_{1}}(-1)^{m_{1}}\left\{\psi_{1}, \psi_{2}\right\}_{\left.i_{\sigma\left(1_{\psi_{2}}\right.}\right)}^{i_{1_{\psi_{2}}}}
\end{aligned}
$$

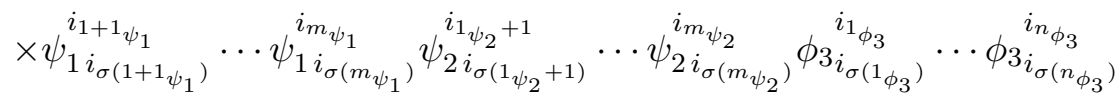

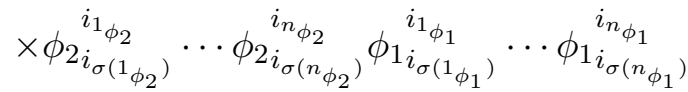

Introduce the notation $\rho_{i}=\sigma(i, 1)$ and rewrite the sum over $S_{n_{T}}$ as a sum over $S_{n_{T}-1}$ and its cosets. The $S_{n_{T}-1}$ subgroup is obtained by restricting to permutations that leave 1 fixed, i.e. $\sigma(1)=1$. The result is

$$
\begin{aligned}
& A=m_{1} m_{2} \sum_{\sigma \in S_{n_{T}-1}} \sum_{i=1}^{n_{T}} \operatorname{Tr}_{(\vec{r}, \vec{s}) \vec{\mu} \vec{\nu}}\left(\Gamma^{(R)}\left(\left[\rho_{i},\left(1,1_{\psi_{2}}\right)\right]\right)\right) \delta_{i_{\rho_{i}(1)}}^{i_{1}}(-1)^{m_{1}}\left\{\psi_{1}, \psi_{2}\right\}_{i_{\rho_{i}\left(\psi_{\psi_{2}}\right)}}^{i_{1_{1}}} \psi_{1 i_{\rho_{i}(2)}}^{i_{2}} \cdots \psi_{1 i_{\rho_{i}\left(m_{1}\right)}}^{i_{m_{1}}}
\end{aligned}
$$

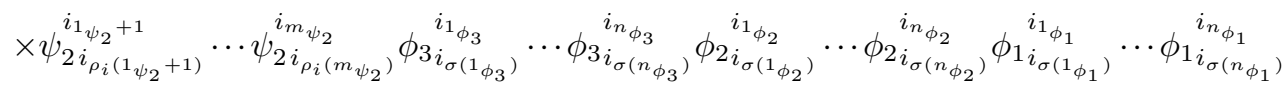

$$
\begin{aligned}
& =m_{1} m_{2} \sum_{\sigma \in S_{n_{T}-1}} \operatorname{Tr}_{(\vec{r}, \vec{s}) \vec{\mu} \vec{\nu}}\left(\Gamma^{(R)}\left(\left[\sigma\left\{N+\sum_{i=1}^{n_{T}}(i, 1)\right\},\left(1,1_{\psi_{2}}\right)\right]\right)\right) \\
& \times(-1)^{m_{1}} \operatorname{Tr}\left(\sigma \cdot \psi_{1}^{\otimes m_{1}-1}\left\{\psi_{1}, \psi_{2}\right\} \psi_{2}^{\otimes m_{2}-1} \phi_{3}{ }^{\otimes n_{3}} \phi_{2}{ }^{\otimes n_{2}} \phi_{1}{ }^{\otimes n_{1}}\right) \\
& =m_{1} m_{2} \sum_{R^{\prime}} c_{R R^{\prime}} \sum_{\sigma \in S_{n_{T}-1}} \operatorname{Tr}_{(\vec{r}, \vec{s}) \vec{\mu} \vec{\nu}}\left(\left[\Gamma^{\left(R^{\prime}\right)}(\sigma), \Gamma^{(R)}\left(\left(1,1_{\psi_{2}}\right)\right)\right]\right) \\
& \times(-1)^{m_{1}} \operatorname{Tr}\left(\sigma \cdot \psi_{1}^{\otimes m_{1}-1}\left\{\psi_{1}, \psi_{2}\right\} \psi_{2}^{\otimes m_{2}-1} \phi_{3}^{\otimes n_{3}} \phi_{2}^{\otimes n_{2}} \phi_{1}^{\otimes n_{1}}\right)
\end{aligned}
$$

We are summing over the subgroup $S_{n_{T}-1}$ of the group $S_{n_{T}}$. After restriction to the subgroup the irreducible representation $R$ of $S_{n_{T}}$ gives all representations $R^{\prime}$ obtained by dropping a single box from $R$, such that the result is still a valid Young diagram. After restricting each $R^{\prime}$ appears exactly once. In moving from the second last to the last line above we use the fact that $\sum_{i=1}^{n_{T}}(i, 1)$ is a Jucys-Murphy element, and the eigenvalues of these elements acting on any state in $R^{\prime}$ is the factor of the box dropped from $R$ to obtain $R^{\prime}$. We denote the factor of this box by $c_{R R^{\prime}}$. Recall that the factor of the box in row $i$ and column $j$ is $N-i+j$. For a discussion with all the details, the reader should consult appendix B of [14]. Now, we can write (recall that $\sigma(1)=1$ )

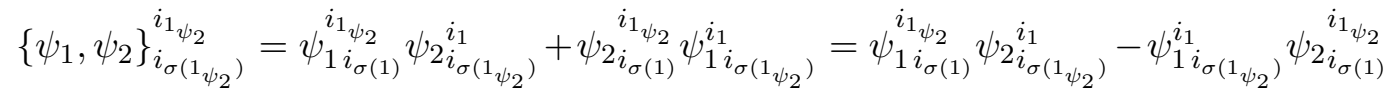

Consequently

$$
\begin{array}{r}
(-1)^{m_{1}} \operatorname{Tr}\left(\sigma \cdot \psi_{1}^{\otimes m_{1}-1}\left\{\psi_{1}, \psi_{2}\right\} \psi_{2}^{\otimes m_{2}-1} \phi_{3}^{\otimes n_{3}} \phi_{2}^{\otimes n_{2}} \phi_{1}^{\otimes n_{1}}\right) \\
=\operatorname{Tr}\left(\left[\left(1,1_{\psi_{2}}\right), \sigma\right] \cdot \psi_{1}^{\otimes m_{1}} \psi_{2}^{\otimes m_{2}} \phi_{3}^{\otimes n_{3}} \phi_{2}^{\otimes n_{2}} \phi_{1}^{\otimes n_{1}}\right)
\end{array}
$$

Thus, we now have

$$
\begin{array}{r}
A=m_{1} m_{2} \sum_{R^{\prime}} c_{R R^{\prime}} \sum_{\sigma \in S_{n_{T}-1}} \operatorname{Tr}_{(\vec{r}, \vec{s}) \vec{\mu} \vec{\nu}}\left(\left[\Gamma^{\left(R^{\prime}\right)}(\sigma), \Gamma^{(R)}\left(\left(1,1_{\psi_{2}}\right)\right)\right]\right) \\
\operatorname{Tr}\left(\left[\left(1,1_{\psi_{2}}\right), \sigma\right] \cdot \psi_{1}^{\otimes m_{1}} \psi_{2}^{\otimes m_{2}} \psi_{1} \phi_{3}^{\otimes n_{3}} \phi_{2}^{\otimes n_{2}} \phi_{1}^{\otimes n_{1}}\right)
\end{array}
$$


At this point use (3.3) to obtain

$$
\begin{aligned}
A= & \sum_{T, \vec{t} \vec{u}) \vec{\alpha} \vec{\beta}} \frac{m_{1} m_{2} d_{T} n_{1} ! n_{2} ! n_{3} ! m_{1} ! m_{2} !}{d_{t_{1}} d_{t_{2}} d_{t_{3}} d_{u_{1}} d_{u_{2}} n_{T} !} \sum_{R^{\prime}} c_{R R^{\prime}} \\
& \sum_{\sigma \in S_{n_{T}-1}} \operatorname{Tr}_{(\vec{r}, \vec{s}) \vec{\mu} \vec{\nu}}\left(\left[\Gamma^{\left(R^{\prime}\right)}(\sigma), \Gamma^{(R)}\left(\left(1,1_{2}\right)\right)\right]\right) \operatorname{Tr}_{(\vec{t} \vec{u}) \vec{\alpha} \vec{\beta}}\left(\left[\Gamma^{(T)}(\sigma), \Gamma^{(R)}\left(\left(1,1_{2}\right)\right)\right]\right) \chi_{T,(\vec{t} \vec{u}) \vec{\beta} \vec{\alpha}}\left(\phi_{i}, \psi_{a}\right)
\end{aligned}
$$

The final step entails using Schur's orthogonality relations to perform the sum over the subgroup. In the basis of normalized restricted Schur polynomials, the result is

$$
\begin{aligned}
& D_{\psi_{1} \psi_{2}} O_{R,(\vec{r} \vec{s}) \vec{\mu} \vec{\nu}}=\sum_{R^{\prime}} \sum_{T,(\vec{t} \vec{u}) \vec{\alpha} \vec{\beta}} \sqrt{c_{R R^{\prime}} c_{T T^{\prime}}} \sqrt{\frac{\text { hooks }_{\vec{r}} \text { hooks }_{\vec{s}} \text { hooks }_{\vec{t}} \text { hooks }_{\vec{u}}}{\text { hooks }_{T} \text { hooks }_{R}}} \frac{m_{1} m_{2} \sqrt{\text { hooks }_{R^{\prime}} \text { hookss }_{T^{\prime}}}}{n_{1} ! n_{2} ! n_{3} ! m_{1} ! m_{2} !} \\
& \quad \times \operatorname{Tr}_{R \oplus T}\left(\left[P_{R,(\vec{r} \vec{s}) \vec{\mu} \vec{\nu}}, \Gamma^{(R)}\left(\left(1,1_{2}\right)\right)\right] I_{R^{\prime} T^{\prime}}\left[P_{T,(\vec{t} \vec{u}) \vec{\alpha} \vec{\beta}}, \Gamma^{(T)}\left(\left(1,1_{2}\right)\right)\right] I_{T^{\prime} R^{\prime}}\right) O_{T,(\vec{t} \vec{u}) \vec{\beta} \vec{\alpha}}\left(\phi_{i}, \psi_{a}\right)
\end{aligned}
$$

where

$$
D_{\psi_{1} \psi_{2}}=\left\{\psi_{1}, \psi_{2}\right\}_{j}^{i}\left(\frac{d}{d \psi_{1 j}^{k}} \frac{d}{d \psi_{2}^{i}}+\frac{d}{d \psi_{2 j}^{k}} \frac{d}{d \psi_{1 k}^{i}}\right)
$$

In writing (3.7) we have introduced the intertwining map $I_{R^{\prime} T^{\prime}}$ which maps from the carrier space of $T^{\prime}$ to the carrier space of $R^{\prime}$. This map vanishes if $R^{\prime}$ and $T^{\prime}$ do not have the same shape, which implies that the above matrix element is non-zero if and only if $R$ and $T$ differ at most, by one box. This map arises from the application of Schur's orthogonality relation, when performing the sum over the $S_{n_{T}-1}$ subgroup.

Using the same procedure, all terms appearing in the dilatation operator can be evaluated. In terms of the index $A=\left\{\phi_{1}, \phi_{2}, \phi_{3}, \psi_{1}, \psi_{2}\right\}$ introduced above, we write $1_{A}=\left\{1_{\phi_{1}}, 1_{\phi_{2}}, 1_{\phi_{3}}, 1_{\psi_{1}}, 1_{\psi_{2}}\right\}$ and $N_{A}=\left\{n_{1}, n_{2}, n_{3}, m_{1}, m_{2}\right\}$. The action of the dilatation operator in the restricted Schur polynomial basis can be summarized as follows

$$
\begin{aligned}
& D O_{R,(\vec{r} \vec{s}) \vec{\mu} \vec{\nu}}\left(\psi_{a}, \phi_{i}\right)=-\frac{2 g_{\mathrm{YM}}^{2}}{(4 \pi)^{2}} \sum_{A>B=1}^{5} \sum_{T,(\vec{t} \vec{u}) \vec{\alpha} \vec{\beta}}\left(\mathcal{M}_{A B}\right)_{R,(\vec{r} \vec{s}) \vec{\mu} \vec{\nu}, T,(\vec{t} \vec{u}) \vec{\alpha} \vec{\beta}} O_{T,(\vec{t} \vec{u}) \vec{\beta} \vec{\alpha}}\left(\psi_{a}, \phi_{i}(3.9)\right. \\
& \left(\mathcal{M}_{A B}\right)_{R,(\vec{r} \vec{s}) \vec{\mu} \vec{\nu}, T,(\vec{u} \vec{u}) \vec{\alpha} \vec{\beta}}=\sum_{R^{\prime}} \sqrt{c_{R R^{\prime}} c_{T T^{\prime}}} \sqrt{\frac{\text { hooks }_{\vec{r}} \operatorname{hooks}_{\vec{s}} \operatorname{hooks}_{\vec{t}} \text { hooks }_{\vec{u}}}{\text { hooks }_{T} \operatorname{hooks}_{R}}} \frac{N_{A} N_{B} \sqrt{\text { hooks }_{R^{\prime}} \text { hooks }_{T^{\prime}}}}{n_{1} ! n_{2} ! n_{3} ! m_{1} ! m_{2} !} \\
& \operatorname{Tr}_{R \oplus T}\left(\left[\Gamma^{(R)}\left(\left(1,1_{A}\right)\right) P_{R,(\vec{r} \vec{s}) \vec{\mu} \vec{\nu}} \Gamma^{(R)}\left(\left(1,1_{A}\right)\right), \Gamma^{(R)}\left(\left(1,1_{B}\right)\right)\right] I_{R^{\prime} T^{\prime}}\right. \\
& \left.\times\left[\Gamma^{(T)}\left(\left(1,1_{A}\right)\right) P_{T,(\vec{t} \vec{u}) \vec{\alpha} \vec{\beta}^{(T)}}\left(\left(1,1_{A}\right)\right), \Gamma^{(T)}\left(\left(1,1_{B}\right)\right)\right] I_{T^{\prime} R^{\prime}}\right)
\end{aligned}
$$

This result is exact in $1 / N$. The fomulas (3.9) and (3.10) give the complete action of the dilatation operator in the $\mathrm{su}(2 \mid 3)$ sector, which is one of the new results of this paper. 


\section{Dilatation operator on Gauss graphs}

In this section we specialize the discussion and focus on operators dual to giant graviton branes. This class of operators can be described using the Gauss graph basis. The basic result we achieve is a rewriting of the matrix elements of the dilatation operator in the Gauss graph basis. Concretely this entails computing the following Fourier transform

$$
\left(M_{A B}\right)_{R, r_{1}, \vec{\sigma}_{1}, T, t_{1}, \vec{\sigma}_{2}}=\sum_{\substack{r_{2}, r_{3}, \vec{s}, \vec{\mu} \overrightarrow{\vec{v}} \\ t_{2}, t_{3}, \vec{u}, \vec{\alpha} \vec{\beta}}} C_{\vec{\mu} \vec{\nu}}^{\left(r_{2}, r_{3}, \vec{s}\right)}\left(\vec{\sigma}_{1}\right) C_{\vec{\alpha} \vec{\beta}}^{\left(t_{2}, t_{3}, \vec{u}\right)}\left(\vec{\sigma}_{2}\right)\left(\mathcal{M}_{A B}\right)_{R,(\vec{r} \vec{s}) \vec{\mu} \vec{\nu}, T,(\vec{t} \vec{u}) \vec{\alpha} \vec{\beta}}
$$

where

$$
C_{\vec{\mu} \vec{\nu}}^{\left(r_{2}, r_{3}, \vec{s}\right)}(\vec{\sigma})=C_{\mu_{1} \nu_{1}}^{\left(r_{2}\right)}\left(\sigma_{\phi_{2}}\right) C_{\mu_{2} \nu_{2}}^{\left(r_{3}\right)}\left(\sigma_{\phi_{3}}\right) \tilde{C}_{\mu_{3} \nu_{3}}^{\left(s_{1}\right)}\left(\sigma_{\psi_{1}}\right) \tilde{C}_{\mu_{4} \nu_{4}}^{\left(s_{2}\right)}\left(\sigma_{\psi_{2}}\right)
$$

Our first task is to simplify the trace

$$
\begin{aligned}
\mathcal{T}_{A B}=\operatorname{Tr}_{R \oplus T}( & {\left[\Gamma^{(R)}\left(\left(1,1_{A}\right)\right) P_{R,(\vec{r} \vec{s}) \vec{\mu} \vec{\nu}} \Gamma^{(R)}\left(\left(1,1_{A}\right)\right), \Gamma^{(R)}\left(\left(1,1_{B}\right)\right)\right] I_{R^{\prime} T^{\prime}} } \\
& \left.\times\left[\Gamma^{(T)}\left(\left(1,1_{A}\right)\right) P_{T,(\vec{t} \vec{u}) \vec{\alpha} \vec{\beta}} \Gamma^{(T)}\left(\left(1,1_{A}\right)\right), \Gamma^{(T)}\left(\left(1,1_{B}\right)\right)\right] I_{T^{\prime} R^{\prime}}\right)
\end{aligned}
$$

which appears in the expression for the term in the one loop dilatation operator that mixes $A$ and $B$ type excitations. Up to this point we have worked at one loop, but to all orders in $1 / N$. We will for the first time start to use some of the simplifications of large $N$ by working in the displaced corners approximation.

We start by introducing a vector space as explained in [22]. Each box associated to an excitation becomes a $p$-dimensional vector in a space $V_{p}$. Excitation boxes belonging to the $i$ th row of $R$ are represented by vectors that have all entries equal to zero except for the $i$ th entry which is 1 . In this way the collection of impurities become a vector in $V_{p}^{\otimes n_{2}+n_{3}+m_{1}+m_{2}}$. To explain the utility of this vector space, recall that each Young diagram $R$ can be labeled to produce a set of Young-Yamanouchi (YY) symbols. Each YY symbol corresponds to a state in the carrier space of irreducible representation $R$. Translating each YY symbol into a vector in $V_{p}^{\otimes n_{2}+n_{3}+m_{1}+m_{2}}$, the action of the symmetric group on $R$ becomes a simple action of permuting vectors in $V_{p}^{\otimes n_{2}+n_{3}+m_{1}+m_{2}}$. For a detailed account of this mathematical framework the reader should consult [32].

The calculations of this section make extensive use of (2.10) which writes the intertwining map used to construct the restricted Schur polynomial as a tensor product with a factor for each species of field. The factor associated to the $\phi_{1}$ field is a projection operator. The factors associated to excitation fields are themselves intertwining maps.

Imagine that $R^{\prime}$ is obtained from $R$ by dropping a box in row $i$ and $T^{\prime}$ from $T$ by dropping a box from row $j$. The corresponding intertwining maps are

$$
I_{R^{\prime} T^{\prime}}=E_{i j}^{(1)}, \quad I_{T^{\prime} R^{\prime}}=E_{j i}^{(1)}
$$

Here the $E_{i j}$ are the usual basis for $\operatorname{GL}(N)$, i.e. $\left(E_{i j}\right)_{a b}=\delta_{i a} \delta_{j b}$. The superscript tells us which factor in the tensor product $E_{i j}$ acts on. To evaluate the traces, write the 
permutations appearing in the above trace (4.3) in terms of the $E_{i j}$ 's

$$
\left(1_{A}, 1_{B}\right)=\sum_{i, j=1}^{p} E_{i j}^{\left(1_{A}\right)} E_{j i}^{\left(1_{B}\right)}
$$

and simplify the product of the E's using the usual algebra

$$
E_{i j} E_{k l}=\delta_{j k} E_{i l}
$$

By the usual rules for the tensor product, Es only multiply with each other if they are in the same slot. Since the trace $\mathcal{T}$ is a product of two commutators, expanding gives four terms. After expanding we have

$$
\begin{aligned}
\mathcal{T}_{A B}= & \left(\operatorname{Tr}_{R \oplus T}\left(\left(1,1_{A}\right) P_{R,(\vec{r} \vec{s}) \vec{\mu} \vec{\nu}}\left(1,1_{A}\right)\left(1,1_{B}\right) I_{R^{\prime} T^{\prime}}\left(1,1_{A}\right) P_{T,(\vec{t} \vec{u}) \vec{\alpha} \vec{\beta}}\left(1,1_{A}\right)\left(1,1_{B}\right) I_{T^{\prime} R^{\prime}}\right)\right. \\
& -\operatorname{Tr}_{R \oplus T}\left(\left(1,1_{B}\right)\left(1,1_{A}\right) P_{R,(\vec{r} \vec{s}) \vec{\mu} \vec{\nu}}\left(1,1_{A}\right) I_{R^{\prime} T^{\prime}}\left(1,1_{A}\right) P_{T,(\vec{t} \vec{u}) \vec{\alpha} \vec{\beta}}\left(1,1_{A}\right)\left(1,1_{B}\right) I_{T^{\prime} R^{\prime}}\right) \\
& -\operatorname{Tr}_{R \oplus T}\left(\left(1,1_{A}\right) P_{R,(\vec{r} \vec{s}) \vec{\mu} \vec{\nu}}\left(1,1_{A}\right)\left(1,1_{B}\right) I_{R^{\prime} T^{\prime}}\left(1,1_{B}\right)\left(1,1_{A}\right) P_{T,(\vec{t} \vec{u}) \vec{\alpha} \vec{\beta}}\left(1,1_{A}\right) I_{T^{\prime} R^{\prime}}\right) \\
& \left.+\operatorname{Tr}_{R \oplus T}\left(\left(1,1_{B}\right)\left(1,1_{A}\right) P_{R,(\vec{r} \vec{s}) \vec{\mu} \vec{\nu}}\left(1,1_{A}\right) I_{R^{\prime} T^{\prime}}\left(1,1_{B}\right)\left(1,1_{A}\right) P_{T,(\vec{t} \vec{u}) \vec{\alpha} \vec{\beta}}\left(1,1_{A}\right) I_{T^{\prime} R^{\prime}}\right)\right)
\end{aligned}
$$

Following the procedure described above we find

$$
\begin{aligned}
\left(1,1_{A}\right)\left(1,1_{B}\right) I_{R^{\prime} T^{\prime}}\left(1,1_{A}\right) & =\left(1,1_{A}\right)\left(1,1_{B}\right) E_{i j}^{(1)}\left(1,1_{A}\right)=\left(1_{A}, 1_{B}\right) E_{i j}^{\left(1_{A}\right)} \\
\left(1,1_{A}\right) I_{R^{\prime} T^{\prime}}\left(1,1_{A}\right) & =\left(1,1_{A}\right) E_{i j}^{(1)}\left(1,1_{A}\right)=E_{i j}^{\left(1_{A}\right)} \\
\left(1,1_{A}\right)\left(1,1_{B}\right) I_{T^{\prime} R^{\prime}}\left(1,1_{B}\right)\left(1,1_{A}\right) & =\left(1,1_{A}\right)\left(1,1_{B}\right) E_{j i}^{(1)}\left(1,1_{B}\right)\left(1,1_{A}\right)=E_{j i}^{\left(1_{B}\right)}
\end{aligned}
$$

Thus, the trace now simplifies to

$$
\begin{aligned}
\mathcal{T}_{A B}= & \left(\operatorname{Tr}_{R \oplus T}\left(P_{R,(\vec{r} \vec{s}) \vec{\mu} \vec{\nu}} E_{i a}^{\left(1_{B}\right)} E_{a j}^{\left(1_{A}\right)} P_{T,(\vec{t} \vec{u}) \vec{\alpha} \vec{\beta}} E_{j c}^{\left(1_{B}\right)} E_{c i}^{\left(1_{A}\right)}\right)\right. \\
& -\operatorname{Tr}_{R \oplus T}\left(P_{R,(\vec{r} \vec{s}) \vec{\mu} \vec{\nu}} E_{i j}^{\left(1_{A}\right)} P_{T,(\vec{t} \vec{u}) \vec{\alpha} \vec{\beta}} E_{j i}^{\left(1_{B}\right)}\right)-\operatorname{Tr}_{R \oplus T}\left(P_{R,(\vec{r} \vec{s}) \vec{\mu} \vec{\nu}} E_{i j}^{\left(1_{B}\right)} P_{T,(\vec{t} \vec{u}) \vec{\alpha} \vec{\beta}} E_{j i}^{\left(1_{A}\right)}\right) \\
& \left.+\operatorname{Tr}_{R \oplus T}\left(P_{R,(\vec{r} \vec{s}) \vec{\mu} \vec{\nu}} E_{i b}^{\left(1_{A}\right)} E_{b j}^{\left(1_{B}\right)} P_{T,(\vec{t} \vec{u}) \vec{\alpha} \vec{\beta}} E_{j d}^{\left(1_{A}\right)} E_{d i}^{\left(1_{B}\right)}\right)\right)
\end{aligned}
$$

With this simplified expression in hand we can return to evaluating the sums in (4.1).

The terms involving mixing of excitations with $\phi_{1}$ are significantly simpler due to the fact that the projector is simpler. These terms have already been evaluated for bosons in [12] and for fermions in [25]. The result is (we remind the reader that the integers $\left(n_{2}\right)_{i j},\left(n_{3}\right)_{i j}, \cdots$ were defined in the paragraph after the paragraph containing (2.14))

$$
\begin{aligned}
& D_{\phi_{1} \phi_{2}} O_{R, r_{1}}(\vec{\sigma})=\sum_{i>j=1}^{p}\left(n_{2}\right)_{i j} \Delta_{i j} O_{R, r_{1}}(\vec{\sigma}) \\
& D_{\phi_{1} \phi_{3}} O_{R, r_{1}}(\vec{\sigma})=\sum_{i>j=1}^{p}\left(n_{3}\right)_{i j} \Delta_{i j} O_{R, r_{1}}(\vec{\sigma})
\end{aligned}
$$




$$
\begin{aligned}
& D_{\phi_{1} \psi_{1}} O_{R, r_{1}}(\vec{\sigma})=\sum_{i>j=1}^{p}\left(m_{1}\right)_{i j} \Delta_{i j} O_{R, r_{1}}(\vec{\sigma}) \\
& D_{\phi_{1} \psi_{2}} O_{R, r_{1}}(\vec{\sigma})=\sum_{i>j=1}^{p}\left(m_{2}\right)_{i j} \Delta_{i j} O_{R, r_{1}}(\vec{\sigma})
\end{aligned}
$$

The operator $\Delta_{i j}$ is a sum of three terms

$$
\Delta_{i j}=\Delta_{i j}^{+}+\Delta_{i j}^{0}+\Delta_{i j}^{-}
$$

$\Delta_{i j}$ acts only on the $R, r_{1}$ labels of the Gauss graph operator. Denote the row lengths of Young diagram $r$ by $l_{r}$. Young diagram $r_{i j}^{+}$is obtained by removing a box from row $j$ and adding it to row $i$ and $r_{i j}^{-}$is obtained by removing a box from row $i$ and adding it to row $j$. With this notation the action of the terms appearing in $\Delta_{i j}$ are

$$
\begin{aligned}
& \Delta_{i j}^{0} O_{R, r}(\vec{\sigma})=-\left(2 N+l_{r_{i}}+l_{r_{j}}\right) O_{R, r}(\vec{\sigma}) \\
& \Delta_{i j}^{ \pm} O_{R, r}(\vec{\sigma})=\sqrt{\left(N+l_{r_{i}}\right)\left(N+l_{r_{j}}\right)} O_{R_{i j}^{ \pm}, r_{i j}^{ \pm}}(\vec{\sigma})
\end{aligned}
$$

The above result is rather natural. To see this, we recall a few facts from the planar limit. In the planar limit the operator $\operatorname{Tr}\left(Z^{n}\right)$ is an exact eigenstate of the dilatation operator. It is annihilated by the one loop (and in fact higher loops) dilatation operator because it is a half BPS operator and its dimension is protected. The operator $\operatorname{Tr}\left(Y Z^{n-m} Y Z^{m}\right)$ is quarter BPS at zero coupling. When the coupling is turned on, operators with different values of $m$ start to mix and those operators with a non-zero eigenvalue under the one loop dilatation operator are not BPS. Now consider the operators relevant to our study. The Schur polynomials constructed using a single complex field $Z$ are half BPS. After including $Y$ fields the resulting operators (restricted Schur polynomials) start to mix and it is again a linear combination of operators that correspond to eigenstates of the dilatation operator, exactly in line with the discussion of the planar limit. A semi-classical state, of definite energy, in the gravity dual is a linear combination of restricted Schur polynomials, labeled by Young diagrams $R$ that do not all have the same shape. The different shapes appearing in the linear combination have row lengths that differ by order 1 numbers. These (as usual) are not important for the dual semi-classical physics. Indeed, the size, angular momentum and position of the giant graviton depends only on the length of the row corresponding to the giant gravuiton, divided by $N$. Consequently, order 1 corrections to row lengths do not change the semi-classical state.

Now consider the contributions $D_{\hat{A} \hat{B}}$ to the dilatation operator that describe the mixing of the excitation fields. These terms share the same structure, so we can carry the discussion out in generality. Using the results of [38], we obtain the following expression

$$
D_{\hat{A} \hat{B}} \hat{O}_{R, r_{1}}\left(\vec{\sigma}_{1}\right)=\left(M_{\hat{A} \hat{B}}\right)_{R, r_{1}, \vec{\sigma}_{1} T, t_{1}, \vec{\sigma}_{2}} \hat{O}_{T, t_{1}}\left(\vec{\sigma}_{2}\right)
$$

where

$$
\begin{aligned}
& \left(M_{\hat{A} \hat{B}}\right)_{R, r_{1}, \vec{\sigma}_{1} T, t_{1}, \vec{\sigma}_{2}}=\frac{1}{\sqrt{\left|O_{R, r_{1}}\left(\vec{\sigma}_{1}\right)\right|^{2}\left|O_{T, t_{1}}\left(\vec{\sigma}_{2}\right)\right|^{2}}} \prod_{\hat{C} \neq \hat{A}, \hat{B}} \delta_{\left(\sigma_{\hat{C}}\right)_{1}\left(\sigma_{\hat{C}}\right)_{2}} \times \\
& \sum_{R^{\prime}} \frac{\delta_{R_{i}^{\prime} T_{k}^{\prime}} \delta_{r_{1} t_{1}}}{\left(N_{\hat{A}}-1\right) !\left(N_{\hat{B}}-1\right) !} \sqrt{\frac{c_{R R^{\prime} C_{T T^{\prime}}}}{l_{R_{i}} l_{T_{k}}}} \sum_{\psi_{1} \in S_{N_{\hat{A}}} \times S_{N_{\hat{B}}}} \sum_{\psi_{2} \in S_{N_{\hat{A}^{\prime}}} \times S_{N_{\hat{B}^{\prime}}}}
\end{aligned}
$$




$$
\begin{aligned}
& {\left[\left\langle\vec{N}_{\hat{A}}^{\prime}, \vec{N}_{\hat{B}}^{\prime}\left|\sigma_{2} \psi_{2}^{-1} E_{k i}^{\left(1_{\hat{A}}\right)} \psi_{1}\right| \vec{N}_{\hat{A}}, \vec{N}_{\hat{B}}\right\rangle\left\langle\vec{N}_{\hat{A}}, \vec{N}_{\hat{B}}\left|\sigma_{1}^{-1} \psi_{1}^{-1} E_{i k}^{\left(1_{\hat{B}}\right)} \psi_{2}\right| \vec{N}_{\hat{A}}^{\prime}, \vec{N}_{\hat{B}}^{\prime}\right\rangle\right.} \\
& -\left\langle\vec{N}_{\hat{A}}^{\prime}, \vec{N}_{\hat{B}}^{\prime}\left|\sigma_{2} \psi_{2}^{-1} E_{c i}^{\left(1_{\hat{A}}\right)} E_{k c}^{\left(1_{\hat{B}}\right)} \psi_{1}\right| \vec{N}_{\hat{A}}, \vec{N}_{\hat{B}}\right\rangle\left\langle\vec{N}_{\hat{A}}, \vec{N}_{\hat{B}}\left|\sigma_{1}^{-1} \psi_{1}^{-1} E_{a k}^{\left(1_{\hat{A}}\right)} E_{i a}^{\left(1_{\hat{B}}\right)} \psi_{2}\right| \vec{N}_{\hat{A}}^{\prime}, \vec{N}_{\hat{B}}^{\prime}\right\rangle \\
& -\left\langle\vec{N}_{\hat{A}}^{\prime}, \vec{N}_{\hat{B}}^{\prime}\left|\sigma_{2} \psi_{2}^{-1} E_{k c}^{\left(1_{\hat{A}}\right)} E_{c i}^{\left(1_{\hat{B}}\right)} \psi_{1}\right| \vec{N}_{\hat{A}}, \vec{N}_{\hat{B}}\right\rangle\left\langle\vec{N}_{\hat{A}}, \vec{N}_{\hat{B}}\left|\sigma_{1}^{-1} \psi_{1}^{-1} E_{i a}^{\left(1_{\hat{A}}\right)} E_{a k}^{\left(1_{\hat{B}}\right)} \psi_{2}\right| \vec{N}_{\hat{A}}^{\prime}, \vec{N}_{\hat{B}}^{\prime}\right\rangle \\
& \left.+\left\langle\vec{N}_{\hat{A}}^{\prime}, \vec{N}_{\hat{B}}^{\prime}\left|\sigma_{2} \psi_{2}^{-1} E_{k i}^{\left(1_{\hat{B}}\right)} \psi_{1}\right| \vec{N}_{\hat{A}}, \vec{N}_{\hat{B}}\right\rangle\left\langle\vec{N}_{\hat{A}}, \vec{N}_{\hat{B}}\left|\sigma_{1}^{-1} \psi_{1}^{-1} E_{i k}^{\left(1_{\hat{A}}\right)} \psi_{2}\right| \vec{N}_{\hat{A}}^{\prime}, \vec{N}_{\hat{B}}^{\prime}\right\rangle\right]
\end{aligned}
$$

Recall that the Gauss graph operators $\hat{O}_{R, r}\left(\vec{\sigma}_{1}\right)$ are normalized to have a unit two point function. The delta function on the first line of the above expression vanishes if the graphs of the excitations that are not mixing are not equal i.e. $\delta_{\left(\sigma_{\hat{C}}\right)_{1}\left(\sigma_{\hat{C}}\right)_{2}}=1$ as long as $\left(\sigma_{\hat{C}}\right)_{1}$ and $\left(\sigma_{\hat{C}}\right)_{2}$ correspond to the same double coset element. The permutations $\sigma_{1}$ and $\sigma_{2}$ appearing in the above summand stand for the outer product of two permutations. Dropping the subscript for now, we can write $\sigma=\sigma_{\hat{A}} \circ \sigma_{\hat{B}}$ where $(\sigma$ should not be confused with $\vec{\sigma}$ )

$$
\sigma_{\hat{A}} \in H_{\vec{N}_{\hat{A}}} \backslash S_{N_{\hat{A}}} / H_{\vec{N}_{\hat{A}}} \quad \sigma_{\hat{B}} \in H_{\vec{N}_{\hat{B}}} \backslash S_{N_{\hat{B}}} / H_{\vec{N}_{\hat{B}}}
$$

We will also write this as

$$
\sigma \in H \backslash S_{N_{\hat{A}}} \times S_{N_{\hat{B}}} / H
$$

where $H=H_{\vec{N}_{\hat{A}}} \times H_{\vec{N}_{\hat{B}}}$. We use $H_{1}$ for the symmetry group of $\sigma_{1}$ and $H_{2}$ for $\sigma_{2}$. We use $N_{\hat{A}}, N_{\hat{B}}$ for $\sigma_{1}$ and $N_{\hat{A}}^{\prime}, N_{\hat{B}}^{\prime}$ for $\sigma_{2}$ and so on. Some of this is simply for clarity: indeed, we always have $N_{\hat{A}}=N_{\hat{A}}^{\prime}$ and $N_{\hat{B}}=N_{\hat{B}}^{\prime}$, but in general, $\vec{N}_{\hat{A}} \neq \vec{N}_{\hat{A}}^{\prime}$ and $\vec{N}_{\hat{B}} \neq \vec{N}_{\hat{B}}^{\prime}$. Said differently, the number of $A$ and $B$ fields in the two operators are equal, but they may not belong to the same rows in $R$. We need to introduce the vectors $\left(\vec{v}_{i}\right)_{a}=\delta_{i a}$ which form a basis for $V_{p}$. The vector $\left|\vec{N}_{\hat{A}}, \vec{N}_{\hat{B}}\right\rangle$ is defined as follows

$$
\left|\vec{N}_{\hat{A}}, \vec{N}_{\hat{B}}\right\rangle=\left|\vec{N}_{\hat{A}}\right\rangle \otimes\left|\vec{N}_{\hat{B}}\right\rangle
$$

where for any $p$ dimensional vector $\vec{k}$ we have

$$
|\vec{k}\rangle=\left(\vec{v}_{1}\right)^{\otimes k_{1}} \otimes \cdots \otimes\left(\vec{v}_{p}\right)^{\otimes k_{p}}
$$

With this notation in hand, we can now evaluate the sums over $\psi_{1}$ and $\psi_{2}$ in (4.14). Consider the term

$$
T_{1}=\sum_{\substack{\psi_{1} \in S_{N_{\hat{A}}} \times S_{N_{\hat{B}}} \\ \psi_{2} \in S_{N_{\hat{A}}^{\prime}}^{\prime} \times S_{N_{\hat{B}}}^{\prime}}}\left\langle\vec{N}_{\hat{A}}^{\prime}, \vec{N}_{\hat{B}}^{\prime}\left|\sigma_{2} \psi_{2}^{-1} E_{k i}^{\left(1 \hat{A}_{\hat{A}}\right)} \psi_{1}\right| \vec{N}_{\hat{A}}, \vec{N}_{\hat{B}}\right\rangle\left\langle\vec{N}_{\hat{A}}, \vec{N}_{\hat{B}}\left|\sigma_{1}^{-1} \psi_{1}^{-1} E_{i k}^{\left(1_{\hat{B}}\right)} \psi_{2}\right| \vec{N}_{\hat{A}}^{\prime}, \vec{N}_{\hat{B}}^{\prime}\right\rangle
$$

The dependence on the permutations $\sigma_{1}, \sigma_{2}$ can be simplified with the following change of variables: replace $\psi_{2}$ with $\tilde{\psi}_{2}$ where

$$
\tilde{\psi}_{2}=\psi_{2} \sigma_{2}^{-1} \quad \Rightarrow \quad \tilde{\psi}_{2}^{-1}=\sigma_{2} \psi_{2}^{-1}
$$


After relabeling $\tilde{\psi}_{2} \rightarrow \psi_{2}$ and taking the transpose of the first factor which is a real number, we find

$$
T_{1}=\sum_{\substack{\psi_{1} \in S_{N_{\hat{A}}} \times S_{N_{\hat{B}}} \\ \psi_{2} \in S_{N_{\hat{A}}^{\prime}} \times S_{N_{\hat{B}}}^{\prime}}}\left\langle\vec{N}_{\hat{A}}, \vec{N}_{\hat{B}}\left|\psi_{1}^{-1} E_{i k}^{\left(1_{\hat{A}}\right)} \psi_{2}\right| \vec{N}_{\hat{A}}^{\prime}, \vec{N}_{\hat{B}}^{\prime}\right\rangle\left\langle\vec{N}_{\hat{A}}, \vec{N}_{\hat{B}}\left|\sigma_{1}^{-1} \psi_{1}^{-1} E_{i k}^{\left(1_{\hat{B}}\right)} \psi_{2} \sigma_{2}\right| \vec{N}_{\hat{A}}^{\prime}, \vec{N}_{\hat{B}}^{\prime}\right\rangle
$$

If $i \neq k$, the first matrix element in the summand is only non-vanishing if $\vec{N}_{\hat{A}} \neq \vec{N}_{\hat{A}}^{\prime}$ and $\vec{N}_{\hat{B}}=\vec{N}_{\hat{B}}^{\prime}$, while the second matrix element is only non-vanishing if $\vec{N}_{\hat{A}}=\vec{N}_{\hat{A}}^{\prime}$ and $\vec{N}_{\hat{B}} \neq \vec{N}_{\hat{B}}^{\prime}$. Thus, $T_{1}$ vanishes for $i \neq k$, indicated explicitly as follows

$$
T_{1}=\delta_{i k} \sum_{\psi_{1}, \psi_{2} \in S_{N_{\hat{A}}} \times S_{N_{\hat{B}}}}\left\langle\vec{N}_{\hat{A}}, \vec{N}_{\hat{B}}\left|\psi_{1}^{-1} E_{i i}^{\left(1_{\hat{A}}\right)} \psi_{2}\right| \vec{N}_{\hat{A}}, \vec{N}_{\hat{B}}\right\rangle\left\langle\vec{N}_{\hat{A}}, \vec{N}_{\hat{B}}\left|\sigma_{1}^{-1} \psi_{1}^{-1} E_{i i}^{\left(1_{\hat{B}}\right)} \psi_{2} \sigma_{2}\right| \vec{N}_{\hat{A}}, \vec{N}_{\hat{B}}\right\rangle
$$

It makes sense to split the trace up as follows

$$
\begin{aligned}
T_{1}= & \delta_{i k} \sum_{\psi_{1}, \psi_{2} \in S_{N_{\hat{A}}}}\left\langle\vec{N}_{\hat{A}}\left|\psi_{1}^{-1} E_{i i}^{\left(1_{\hat{A}}\right)} \psi_{2}\right| \vec{N}_{\hat{A}}\right\rangle\left\langle\vec{N}_{\hat{A}}\left|\left(\sigma_{\hat{A}}\right)_{1}^{-1} \psi_{1}^{-1} \psi_{2}\left(\sigma_{\hat{A}}\right)_{2}\right| \vec{N}_{\hat{A}}\right\rangle \\
& \times \sum_{\psi_{1}, \psi_{2} \in S_{N_{\hat{B}}}}\left\langle\vec{N}_{\hat{B}}\left|\psi_{1}^{-1} \psi_{2}\right| \vec{N}_{\hat{B}}\right\rangle\left\langle\vec{N}_{\hat{B}}\left|\left(\sigma_{\hat{B}}\right)_{1}^{-1} \psi_{1}^{-1} E_{i i}^{\left(1_{\hat{B}}\right)} \psi_{2}\left(\sigma_{\hat{B}}\right)_{2}\right| \vec{N}_{\hat{B}}\right\rangle
\end{aligned}
$$

Using the easily verified identity

$$
\left\langle\vec{N}\left|\psi_{1}^{-1} \psi_{2}\right| \vec{N}\right\rangle=\sum_{\rho \in H_{\vec{N}}} \delta\left(\rho \psi_{1}^{-1} \psi_{2}\right)
$$

as well as $E_{i j}^{(a)} \psi_{2}=\psi_{2} E_{i j}^{\left(\psi_{2}^{-1}(a)\right)}$ we find

$$
\begin{aligned}
T_{1}= & \delta_{i k} \sum_{\psi_{2} \in S_{N_{\hat{A}}}} \sum_{\gamma \in H_{\vec{N}_{\hat{A}}}}\left\langle\vec{N}_{\hat{A}}\left|\left(\sigma_{\hat{A}}\right) \gamma^{-1}\left(\sigma_{\hat{A}}\right)_{2}^{-1} E_{i i}^{\left(\psi_{2}^{-1}\left(1_{\hat{A}}\right)\right)}\right| \vec{N}_{\hat{A}}\right\rangle \\
& \times \sum_{\psi_{2} \in S_{N_{\hat{B}}}} \sum_{\rho \in H_{\vec{N}_{\hat{B}}}}\left\langle\vec{N}_{\hat{B}}\left|\left(\sigma_{\hat{B}}\right)_{1}^{-1} \rho^{-1} E_{i i}^{\left(\psi_{2}^{-1}\left(1_{\hat{B}}\right)\right)}\left(\sigma_{\hat{B}}\right)_{2}\right| \vec{N}_{\hat{B}}\right\rangle \\
= & \delta_{i k} \sum_{\psi_{2} \in S_{N_{\hat{A}}}} \sum_{l \in S_{i}^{\hat{A}}} \delta\left(\psi_{2}^{-1}\left(1_{\hat{A}}\right), l\right) \sum_{\gamma_{1}, \gamma_{2} \in H_{\vec{N}_{\hat{A}}}} \delta\left(\left(\sigma_{\hat{A}}\right)_{1} \gamma^{-1}\left(\sigma_{\hat{A}}\right)_{2}^{-1}\right) \\
& \times \sum_{\psi_{2} \in S_{N_{\hat{B}}}} \sum_{l \in S_{i}^{\hat{B}}} \delta\left(\psi_{2}^{-1}\left(1_{\hat{B}}\right), l\right) \sum_{\gamma_{1}, \gamma_{2} \in H_{\vec{N}_{\hat{B}}}} \delta\left(\left(\sigma_{\hat{B}}\right)_{1} \gamma^{-1}\left(\sigma_{\hat{B}}\right)_{2}^{-1}\right) \\
= & \delta_{i k}\left(N_{\hat{A}}-1\right) !\left(N_{\hat{A}}^{\sigma_{1}}\right)_{i} \sum_{\gamma_{1}, \gamma_{2} \in H_{\vec{N}_{\hat{A}}}} \delta\left(\left(\sigma_{\hat{A}}\right)_{1} \gamma^{-1}\left(\sigma_{\hat{A}}\right)_{2}^{-1}\right) \\
& \times\left(N_{\hat{B}}-1\right) !\left(N_{\hat{B}}^{\sigma_{1}}\right)_{i} \sum_{\gamma_{1}, \gamma_{2} \in H_{\vec{N}_{\hat{B}}}} \delta\left(\left(\sigma_{\hat{B}}\right)_{1} \gamma^{-1}\left(\sigma_{\hat{B}}\right)_{2}^{-1}\right)
\end{aligned}
$$

and where $S_{i}^{\hat{A}}$ are the slots in $\left|\vec{N}_{\hat{A}}\right\rangle$ occupied by $\vec{v}_{i}$ and $S_{i}^{\hat{B}}$ are the slots in $\left|\vec{N}_{\hat{B}}\right\rangle$ occupied by $\vec{v}_{i}$. We can write this as

$$
T_{1}=\delta_{i k}\left(N_{\hat{A}}-1\right) !\left(N_{\hat{B}}-1\right) !\left(\vec{N}_{\hat{A}}\right)_{i}\left(\vec{N}_{\hat{B}}\right)_{i} \sum_{h_{1}, h_{2} \in H_{2}} \delta\left(\sigma_{1}^{-1} h_{1}^{-1} \sigma_{2} h_{2}\right)
$$


Here $\left(\vec{N}_{\hat{A}}\right)_{i}$ and $\left(\vec{N}_{\hat{B}}\right)_{i}$ count the number of edges ending on node $i$, or equivalently the number of excitations of $\hat{A} / \hat{B}$ living in row $i$. We could, for example, write

$$
\begin{aligned}
\left(\vec{N}_{\hat{A}}\right)_{i} & =\sum_{k \neq i}\left(N_{\hat{A}}\right)_{k \rightarrow i}+\left(N_{\hat{A}}\right)_{i i} \\
& =\sum_{k \neq i}\left(N_{\hat{A}}\right)_{i \rightarrow k}+\left(N_{\hat{A}}\right)_{i i}
\end{aligned}
$$

where the second equality uses the constraints implied by the Gauss Law.

We also need to consider the term

$$
\begin{aligned}
T_{4}= & \sum_{\substack{\psi_{1} \in S_{N_{\hat{A}}} \times S_{N_{\hat{B}}} \\
\psi_{2} \in S_{N_{\hat{A}}^{\prime} \times S_{N_{\hat{B}}^{\prime}}}}}\left\langle\vec{N}_{\hat{A}}^{\prime}, \vec{N}_{\hat{B}}^{\prime}\left|\sigma_{2} \psi_{2}^{-1} E_{k i}^{\left(1_{\hat{B}}\right)} \psi_{1}\right| \vec{N}_{\hat{A}}, \vec{N}_{\hat{B}}\right\rangle\left\langle\vec{N}_{\hat{A}}, \vec{N}_{\hat{B}}\left|\sigma_{1}^{-1} \psi_{1}^{-1} E_{i k}^{\left(1_{\hat{A}}\right)} \psi_{2}\right| \vec{N}_{\hat{A}}^{\prime}, \vec{N}_{\hat{B}}^{\prime}\right\rangle \\
= & \sum_{\substack{\psi_{1} \in S_{N_{\hat{A}}} \times S_{N_{\hat{B}}} \\
\psi_{2} \in S_{N_{\hat{A}}^{\prime}}^{\prime} \times S_{N_{\hat{B}}}}}\left\langle\vec{N}_{\hat{A}}, \vec{N}_{\hat{B}}\left|\sigma_{1}^{-1} \psi_{1}^{-1} E_{i k}^{(1 \hat{A})} \psi_{2}\right| \vec{N}_{\hat{A}}^{\prime}, \vec{N}_{\hat{B}}^{\prime}\right\rangle\left\langle\vec{N}_{\hat{A}}, \vec{N}_{\hat{B}}\left|\psi_{1}^{-1} E_{i k}^{\left(1_{\hat{B}}\right)} \psi_{2} \sigma_{2}^{-1}\right| \vec{N}_{\hat{A}}^{\prime}, \vec{N}_{\hat{B}}^{\prime}\right\rangle
\end{aligned}
$$

Changing variables $\psi_{1}^{-1} \rightarrow \sigma_{1}^{-1} \psi_{1}^{-1}$ shows that $T_{4}=T_{1}$ and hence

$$
T_{1}+T_{4}=2 \delta_{i k}\left(N_{\hat{A}}-1\right) !\left(N_{\hat{B}}-1\right) !\left(\vec{N}_{\hat{A}}\right)_{i}\left(\vec{N}_{\hat{B}}\right)_{i} \sum_{h_{1}, h_{2} \in H_{2}} \delta\left(\sigma_{1}^{-1} h_{1}^{-1} \sigma_{2} h_{2}\right)
$$

The next sum we consider is

$$
T_{2}=\sum_{\substack{\psi_{1} \in S_{N_{\hat{A}}} \times S_{N_{\hat{B}}} \\ \psi_{2} \in S_{N_{\hat{A}}^{\prime}} \times S_{N_{\hat{B}}^{\prime}}}}\left\langle\vec{N}_{\hat{A}}^{\prime}, \vec{N}_{\hat{B}}^{\prime}\left|\sigma_{2} \psi_{2}^{-1} E_{c i}^{\left(1_{\hat{A}}\right)} E_{k c}^{\left(1_{\hat{B}}\right)} \psi_{1}\right| \vec{N}_{\hat{A}}, \vec{N}_{\hat{B}}\right\rangle\left\langle\vec{N}_{\hat{A}}, \vec{N}_{\hat{B}}\left|\sigma_{1}^{-1} \psi_{1}^{-1} E_{a k}^{\left(1_{\hat{A}}\right)} E_{i a}^{\left(1_{\hat{B}}\right)} \psi_{2}\right| \vec{N}_{\hat{A}}^{\prime}, \vec{N}_{\hat{B}}^{\prime}\right\rangle
$$

Changing variables $\psi_{2}^{-1} \rightarrow \tilde{\psi}_{2}^{-1}$ with

$$
\tilde{\psi}_{2}^{-1}=\sigma_{2} \psi_{2}^{-1} \quad \Rightarrow \quad \tilde{\psi}_{2}=\psi_{2} \sigma_{2}^{-1}
$$

the sum becomes

$$
\begin{aligned}
T_{2}= & \sum_{\substack{\psi_{1} \in S_{N_{\hat{A}}} \times S_{N_{\hat{B}}} \\
\psi_{2} \in S_{N_{\hat{A}}^{\prime}} \times S_{N_{\hat{B}}^{\prime}}}}\left\langle\vec{N}_{\hat{A}}^{\prime}, \vec{N}_{\hat{B}}^{\prime}\left|\psi_{2}^{-1} E_{c i}^{\left(1_{\hat{A}}\right)} E_{k c}^{\left(1_{\hat{B}}\right)} \psi_{1}\right| \vec{N}_{\hat{A}}, \vec{N}_{\hat{B}}\right\rangle \\
& \times\left\langle\vec{N}_{\hat{A}}, \vec{N}_{\hat{B}}\left|\sigma_{1}^{-1} \psi_{1}^{-1} E_{a k}^{(1 \hat{A})} E_{i a}^{\left(1_{\hat{B}}\right)} \psi_{2} \sigma_{2}\right| \vec{N}_{\hat{A}}^{\prime}, \vec{N}_{\hat{B}}^{\prime}\right\rangle \\
= & \sum_{\substack{\psi_{1} \in S_{N_{\hat{A}}} \times S_{N_{\hat{B}}} \\
\psi_{2} \in S_{N_{\hat{A}}^{\prime}} \times S_{N_{\hat{B}}}}}\left\langle\vec{N}_{\hat{A}}^{\prime}, \vec{N}_{\hat{B}}^{\prime}\left|\psi_{2}^{-1} \psi_{1} E_{c i}^{\psi_{1}^{-1}\left(1_{\hat{A}}\right)} E_{k c}^{\psi_{1}^{-1}\left(1_{\hat{B}}\right)}\right| \vec{N}_{\hat{A}}, \vec{N}_{\hat{B}}\right\rangle \\
& \times\left\langle\vec{N}_{\hat{A}}, \vec{N}_{\hat{B}}\left|\sigma_{1}^{-1} E_{a k}^{\psi_{1}^{-1}\left(1_{\hat{A}}\right)} E_{i a}^{\psi_{1}^{-1}\left(1_{\hat{B}}\right)} \psi_{1}^{-1} \psi_{2} \sigma_{2}\right| \vec{N}_{\hat{A}}^{\prime}, \vec{N}_{\hat{B}}^{\prime}\right\rangle
\end{aligned}
$$


Change variables $\psi_{2} \rightarrow \rho$ with $\rho=\psi_{1}^{-1} \psi_{2}$ and relabel $\rho \rightarrow \psi_{2}$ to find

$$
\begin{aligned}
T_{2}= & \sum_{\substack{\psi_{1} \in S_{N_{\hat{A}}} \times S_{N_{\hat{B}}} \\
\psi_{2} \in S_{N_{\hat{A}}^{\prime}} \times S_{N_{\hat{B}}^{\prime}}}}\left\langle\vec{N}_{\hat{A}}^{\prime}, \vec{N}_{\hat{B}}^{\prime}\left|\psi_{2}^{-1} E_{c i}^{\psi_{1}^{-1}\left(1_{\hat{A}}\right)} E_{k c}^{\psi_{1}^{-1}\left(1_{\hat{B}}\right)}\right| \vec{N}_{\hat{A}}, \vec{N}_{\hat{B}}\right\rangle \\
& \times\left\langle\vec{N}_{\hat{A}}, \vec{N}_{\hat{B}}\left|\sigma_{1}^{-1} E_{a k}^{\psi_{1}^{-1}\left(1_{\hat{A}}\right)} E_{i a}^{\psi_{1}^{-1}\left(1_{\hat{B}}\right)} \psi_{2} \sigma_{2}\right| \vec{N}_{\hat{A}}^{\prime}, \vec{N}_{\hat{B}}^{\prime}\right\rangle
\end{aligned}
$$

Recall that $\vec{v}_{b}$ denotes the $p$ dimensional vector with all entries zero except the $b$ th entry, which is 1 . For a non-zero contribution, the factor on the first line above requires that

$$
\begin{aligned}
& \vec{N}_{\hat{A}}-\vec{v}_{i}+\vec{v}_{c}=\vec{N}_{\hat{A}}^{\prime} \\
& \vec{N}_{\hat{B}}-\vec{v}_{c}+\vec{v}_{k}=\vec{N}_{\hat{B}}^{\prime}
\end{aligned}
$$

and the factor on the second line above requires

$$
\begin{aligned}
& \vec{N}_{\hat{B}}-\vec{v}_{i}+\vec{v}_{a}=\vec{N}_{\hat{B}}^{\prime} \\
& \vec{N}_{\hat{A}}-\vec{v}_{a}+\vec{v}_{k}=\vec{N}_{\hat{A}}^{\prime}
\end{aligned}
$$

There are two solutions:

Case 1: $\vec{v}_{c}=\vec{v}_{i}$ and $\vec{v}_{a}=\vec{v}_{k}$. In this case $\vec{N}_{\hat{A}}=\vec{N}_{\hat{A}}^{\prime}$ and $\vec{N}_{\hat{B}}-\vec{v}_{i}+\vec{v}_{k}=\vec{N}_{\hat{B}}^{\prime}$.

Case 2: $\vec{v}_{c}=\vec{v}_{k}$ and $\vec{v}_{a}=\vec{v}_{i}$. In this case $\vec{N}_{\hat{B}}=\vec{N}_{\hat{B}}^{\prime}$ and $\vec{N}_{\hat{A}}-\vec{v}_{i}+\vec{v}_{k}=\vec{N}_{\hat{A}}^{\prime}$.

The analysis for case 1 is as follows

$$
\begin{aligned}
T_{2}= & \sum_{\begin{array}{c}
\psi_{1} \in S_{N_{\hat{A}}} \times S_{N_{\hat{B}}} \\
\psi_{2} \in S_{N_{\hat{A}}} \times S_{N_{\hat{B}}^{\prime}}
\end{array}}\left\langle\vec{N}_{\hat{A}}, \vec{N}_{\hat{B}}^{\prime}\left|\psi_{2}^{-1} E_{i i}^{\psi_{1}^{-1}\left(1_{\hat{A}}\right)} E_{k i}^{\psi_{1}^{-1}\left(1_{\hat{B}}\right)}\right| \vec{N}_{\hat{A}}, \vec{N}_{\hat{B}}\right\rangle \\
= & \times\left\langle\vec{N}_{\hat{A}}, \vec{N}_{\hat{B}}\left|\sigma_{1}^{-1} E_{k k}^{\psi_{1}^{-1}\left(1_{\hat{A}}\right)} E_{i k}^{\psi_{1}^{-1}\left(1_{\hat{B}}\right)} \psi_{2} \sigma_{2}\right| \vec{N}_{\hat{A}}, \vec{N}_{\hat{B}}^{\prime}\right\rangle \\
& \quad \times \sum_{\psi_{2} \in S_{N_{\hat{A}}} \times S_{N_{\hat{B}}^{\prime}}}\left\langle\vec{N}_{\hat{A}}, \vec{N}_{\hat{B}}^{\prime}\left|\psi_{2}^{-1}\right| \vec{N}_{\hat{A}}, \vec{N}_{\hat{B}}^{\prime}\right\rangle\left\langle\vec{N}_{\hat{A}}, \vec{N}_{\hat{B}}^{\prime}\left|\sigma_{1}^{-1} \psi_{2} \sigma_{2}\right| \vec{N}_{\hat{A}}, \vec{N}_{\hat{B}}^{\prime}\right\rangle \\
= & \left(N_{\hat{A}}-1\right) !\left(N_{\hat{B}}-1\right) !\left(N_{\hat{A}}\right)_{i \rightarrow k}\left(N_{\hat{B}}\right)_{i i} \sum_{\psi_{2} \in S_{N_{\hat{A}}} \times S_{N_{\hat{B}}^{\prime}} h_{1}, h_{2} \in H_{2}} \delta\left(\psi_{2}^{-1} h_{1}\right) \delta\left(\sigma_{1}^{-1} \psi_{2} \sigma_{2} h_{2}\right) \\
= & \left(N_{\hat{A}}-1\right) !\left(N_{\hat{B}}-1\right) !\left(N_{\hat{A}}\right)_{i \rightarrow k}\left(N_{\hat{B}}\right)_{i i} \sum_{h_{1}, h_{2} \in H_{2}} \delta\left(\sigma_{1}^{-1} h_{1} \sigma_{2} h_{2}\right)
\end{aligned}
$$

A few comments are in order. The operator $E_{i i}^{\psi_{1}^{-1}\left(1_{\hat{A}}\right)}$ acts directly on $\left|\vec{N}_{\hat{A}}\right\rangle$ in the first line above, while the operator $E_{k k}^{\psi_{1}^{-1}\left(1_{A}\right)}$ acts on $\sigma_{1}|\vec{A}\rangle$ in the second line. This is only non-zero for strings stretching from $i$ to $k$. The factor $E_{k i}^{\psi_{1}^{-1}\left(1_{B}\right)}$ removes a closed loop from node $i$ in $\sigma_{1}$ and moves it to node $k$ in $\sigma_{2}$. This term allows edges that have both endpoints on 
a single node to hop between nodes. Note that $\vec{N}_{\hat{A}}=\vec{N}_{\hat{A}}^{\prime}$, but $\vec{N}_{\hat{B}} \neq \vec{N}_{\hat{B}}^{\prime}$. The final inner products are for vectors $\left|\vec{N}_{\hat{A}}^{\prime}, \vec{N}_{\hat{B}}^{\prime}\right\rangle$ and that is why we land up summing over $H_{2}$. If we first do the sum over $\psi_{2}$ and then the sum over $\psi_{1}$, we find that

$$
T_{2}=\left(N_{\hat{A}}-1\right) !\left(N_{\hat{B}}-1\right) !\left(N_{\hat{A}}^{\prime}\right)_{k \rightarrow i}\left(N_{\hat{B}}^{\prime}\right)_{k k} \sum_{h_{1}, h_{2} \in H_{1}} \delta\left(\sigma_{1}^{-1} h_{1} \sigma_{2} h_{2}\right)
$$

It is straightforward to verify the equivalence of (4.28) and (4.29). We now turn to the analysis for case 2 . The analysis proceeds along the same lines as for case 1 . The sum we need to perform is

$$
\begin{aligned}
& T_{2}=\sum_{\substack{\psi_{1} \in S_{N_{\hat{A}}} \times S_{N_{\hat{B}}} \\
\psi_{2} \in S_{N_{\hat{A}}^{\prime}} \times S_{N_{\hat{B}}}}}\left\langle\vec{N}_{\hat{A}}^{\prime}, \vec{N}_{\hat{B}}\left|\psi_{2}^{-1} E_{k i}^{\psi_{1}^{-1}\left(1_{\hat{A}}\right)} E_{k k}^{\psi_{1}^{-1}\left(1_{\hat{B}}\right)}\right| \vec{N}_{\hat{A}}, \vec{N}_{\hat{B}}\right\rangle \\
& \times\left\langle\vec{N}_{\hat{A}}, \vec{N}_{\hat{B}}\left|\sigma_{1}^{-1} E_{i k}^{\psi_{1}^{-1}\left(1_{\hat{A}}\right)} E_{i i}^{\psi_{1}^{-1}\left(1_{\hat{B}}\right)} \psi_{2} \sigma_{2}\right| \vec{N}_{\hat{A}}^{\prime}, \vec{N}_{\hat{B}}\right\rangle \\
& =\left(N_{\hat{A}}-1\right) !\left(N_{\hat{B}}-1\right) !\left(N_{\hat{A}}\right)_{i i}\left(N_{\hat{B}}\right)_{k \rightarrow i} \\
& \sum_{\psi_{2} \in S_{N_{\hat{A}}^{\prime}} \times S_{N_{\hat{B}}}}\left\langle\vec{N}_{\hat{A}}^{\prime}, \vec{N}_{\hat{B}}\left|\psi_{2}^{-1}\right| \vec{N}_{\hat{A}}, \vec{N}_{\hat{B}}^{\prime}\right\rangle\left\langle\vec{N}_{\hat{A}}^{\prime}, \vec{N}_{\hat{B}}\left|\sigma_{1}^{-1} \psi_{2} \sigma_{2}\right| \vec{N}_{\hat{A}}^{\prime}, \vec{N}_{\hat{B}}\right\rangle \\
& =\left(N_{\hat{A}}-1\right) !\left(N_{\hat{B}}-1\right) !\left(N_{\hat{A}}\right)_{i i}\left(N_{\hat{B}}\right)_{k \rightarrow i} \sum_{\psi_{2} \in S_{N_{\hat{A}}^{\prime}} \times S_{N_{\hat{B}}} h_{1}, h_{2} \in H_{\vec{N}_{\hat{A}}^{\prime}} \times H_{\vec{N}_{\hat{B}}}} \delta\left(\psi_{2}^{-1} h_{1}\right) \delta\left(\sigma_{1}^{-1} \psi_{2} \sigma_{2} h_{2}\right) \\
& =\left(N_{\hat{A}}-1\right) !\left(N_{\hat{B}}-1\right) !\left(N_{\hat{A}}\right)_{i i}\left(N_{\hat{B}}\right)_{k \rightarrow i} \sum_{h_{1}, h_{2} \in H_{2}} \delta\left(\sigma_{1}^{-1} h_{1} \sigma_{2} h_{2}\right)
\end{aligned}
$$

Thus, for $T_{2}$ we find

$$
T_{2}=\left(N_{\hat{A}}-1\right) !\left(N_{\hat{B}}-1\right) !\left(\left(N_{\hat{A}}\right)_{i \rightarrow k}\left(N_{\hat{B}}\right)_{i i}+\left(N_{\hat{A}}\right)_{i i}\left(N_{\hat{B}}\right)_{k \rightarrow i}\right) \sum_{h_{1}, h_{2} \in H_{2}} \delta\left(\sigma_{1}^{-1} h_{1} \sigma_{2} h_{2}\right)
$$

which can also be written as

$$
T_{2}=\left(N_{\hat{A}}-1\right) !\left(N_{\hat{B}}-1\right) !\left(\left(N_{\hat{A}}^{\prime}\right)_{k \rightarrow i}\left(N_{\hat{B}}^{\prime}\right)_{k k}+\left(N_{\hat{A}}^{\prime}\right)_{k k}\left(N_{\hat{B}}^{\prime}\right)_{i \rightarrow k}\right) \sum_{h_{1}, h_{2} \in H_{1}} \delta\left(\sigma_{1}^{-1} h_{1} \sigma_{2} h_{2}\right)
$$

A very similar analysis now gives

$$
T_{3}=\left(N_{\hat{A}}-1\right) !\left(N_{\hat{B}}-1\right) !\left(\left(N_{\hat{A}}\right)_{k \rightarrow i}\left(N_{\hat{B}}\right)_{i i}+\left(N_{\hat{A}}\right)_{i i}\left(N_{\hat{B}}\right)_{i \rightarrow k}\right) \sum_{h_{1}, h_{2} \in H_{2}} \delta\left(\sigma_{1}^{-1} h_{1} \sigma_{2} h_{2}\right)
$$

which can also be written as

$$
T_{3}=\left(N_{\hat{A}}-1\right) !\left(N_{\hat{B}}-1\right) !\left(\left(N_{\hat{A}}^{\prime}\right)_{i \rightarrow k}\left(N_{\hat{B}}^{\prime}\right)_{k k}+\left(N_{\hat{A}}^{\prime}\right)_{k k}\left(N_{\hat{B}}^{\prime}\right)_{k \rightarrow i}\right) \sum_{h_{1}, h_{2} \in H_{1}} \delta\left(\sigma_{1}^{-1} h_{1} \sigma_{2} h_{2}\right)
$$


Summing the four contributions, we now obtain a rather simple formula for the matrix elements

$$
\begin{aligned}
& \left(M_{\hat{A} \hat{B}}\right)_{R, r_{1}, \vec{\sigma}_{1} T, t_{1}, \vec{\sigma}_{2}}=\prod_{\hat{C} \neq \hat{A}, \hat{B}} \delta_{\left(\sigma_{\hat{C}}\right)_{1}\left(\sigma_{\hat{C}}\right)_{2}} \sum_{R^{\prime}} \frac{\delta_{r_{1} t_{1}} \delta_{R_{i}^{\prime} T_{k}^{\prime}}}{\sqrt{\left|O_{R, r_{1}}\left(\vec{\sigma}_{1}\right)\right|^{2}\left|O_{T, t_{1}}\left(\vec{\sigma}_{2}\right)\right|^{2}}} \sqrt{\frac{c_{R R^{\prime}} c_{T T^{\prime}}}{l_{R_{i}} l_{T_{k}}}} \\
& \times\left[2 \delta_{i k}\left(N_{\hat{A}}\right)_{i}\left(N_{\hat{B}}\right)_{i}-\left(\left(N_{\hat{A}}\right)_{k i}\left(N_{\hat{B}}\right)_{i i}+\left(N_{\hat{A}}\right)_{i i}\left(N_{\hat{B}}\right)_{i k}\right)\right] \sum_{h_{1}, h_{2} \in H_{2}} \delta\left(\sigma_{1}^{-1} h_{1} \sigma_{2} h_{2}\right)
\end{aligned}
$$

which can also be written as

$$
\begin{aligned}
& \left(M_{\hat{A} \hat{B}}\right)_{R, r_{1}, \vec{\sigma}_{1} T, t_{1}, \vec{\sigma}_{2}}=\prod_{\hat{C} \neq \hat{A}, \hat{B}} \delta_{\left(\sigma_{\hat{C}}\right)_{1}\left(\sigma_{\hat{C}}\right)_{2}} \sum_{R^{\prime}} \frac{\delta_{r_{1} t_{1}} \delta_{R_{i}^{\prime} T_{k}^{\prime}}}{\sqrt{\left|O_{R, r_{1}}\left(\vec{\sigma}_{1}\right)\right|^{2}\left|O_{T, t_{1}}\left(\vec{\sigma}_{2}\right)\right|^{2}}} \sqrt{\frac{c_{R R^{\prime}} c_{T T^{\prime}}}{l_{R_{i}} l_{T_{k}}}} \\
& \times\left[2 \delta_{i k}\left(N_{\hat{A}}^{\prime}\right)_{i}\left(N_{\hat{B}}^{\prime}\right)_{i}-\left(\left(N_{\hat{A}}^{\prime}\right)_{k i}\left(N_{\hat{B}}^{\prime}\right)_{k k}+\left(N_{\hat{A}}^{\prime}\right)_{k k}\left(N_{\hat{B}}^{\prime}\right)_{i k}\right)\right] \sum_{h_{1}, h_{2} \in H_{1}} \delta\left(\sigma_{1}^{-1} h_{1} \sigma_{2} h_{2}\right)
\end{aligned}
$$

Finally, note that the norm of the Gauss graph operator is given by

$$
\left|O_{R, r_{1}}(\vec{\sigma})\right|^{2}=\prod_{\hat{A}=1}^{4} \prod_{i, j=1}^{p}\left(N_{\hat{A}}^{\vec{\sigma}}\right)_{i \rightarrow j} !
$$

The result (4.35) is one of the new results of this paper.

\section{$5 \quad$ Emergent lattice model}

The formula for the dilatation operator in Gauss graph basis has a fascinating structure. There are two types of terms. There are four terms mixing $\phi_{1}$ with the excitations, summarized in (4.10). These terms do not act on the Gauss graph label i.e. operators that mix have the same Gauss graph label, but different $R, r_{1}$ labels. There are also six terms, mixing the excitations, summarized in (4.13) and (4.34). These terms act only on the Gauss graph labels i.e. operators that mix have the same $R, r_{1}$ labels, but different Gauss graph labels. Consequently, these terms can be simultaneously diagonalized.

The mixing between operators with different Gauss graphs is tightly constrained. Recall that edges in the Gauss graph come in four species, one for each excitation $\hat{A}$. The edges are oriented and the number of edges of each species entering each node must match the number of edges of the same species leaving the node. The dilatation operator only mixes Gauss graphs that have the same number of edges of each species. There is an even tighter constraint on the mixing: graphs can only mix if they have exactly the same number, orientation and species of edges stretching between distinct nodes. Consequently, if two operators mix their graphs differ only by the placement of the edges that have both endpoints attached to a single node.

In this section we would like to interpret the dilatation operator as a Hamiltonian acting on the Gauss graph, using ideas first described in [39]. The dynamics is all in the 
closed edges that have both end points attached to a node. We will identify these closed edges as particles hopping on a lattice, with lattice sites given by the nodes of the Gauss graph. Of course, each node in the Gauss graph corresponds to a row in $R$, and each row in $R$ corresponds to a giant graviton brane. In the next section we will show that these closed edges are in fact quanta of the brane worldvolume theory. To obtain the "graph dynamics" we introduce a collection of creation and annihilation operators, one for each species of edge. The matrix elements (4.10) and (4.34) are written entirely in terms of the number of edges appearing in the graph. If we translate each graph into a Fock space state, by interpreting the graph as an occupation number representation of the state, then the number of edges can be written using the usual number operator. The hopping of closed edges between nodes is easily accomplished by destroying an edge at one node and creating it at another.

To proceed, introduce two sets of bosonic oscillator operators, $\left(b_{1}\right)_{i j},\left(\bar{b}_{1}\right)_{i j}$ for $\phi_{2}$ corresponding to $\hat{A}=1$ and $\left(b_{2}\right)_{i j},\left(\bar{b}_{2}\right)_{i j}$ for $\phi_{3}(\hat{A}=2)$, as well as two sets of fermionic oscillator operators, $\left(f_{1}\right)_{i j},\left(\bar{f}_{1}\right)_{i j}$ for $\psi_{1}(\hat{A}=3)$ and $\left(f_{2}\right)_{i j},\left(\bar{f}_{2}\right)_{i j}$ for $\psi_{2}(\hat{A}=4)$. Since we want to create and destroy edges with end points at any two nodes, the indices $i, j$ must range over $1,2, \cdots, p$. Thus, the dynamics is that of $p \times p$ matrices, where we recall that the number of rows in $R$ is $p$. Note that the original theory is based on a gauge theory with $\mathrm{U}(N)$ gauge group and hence it involves $N \times N$ and not $p \times p$ matrices. To refer to the complete collection of bosonic and fermionic oscillators we will use $\left(a_{\hat{A}}\right)_{i j},\left(\bar{a}_{\hat{A}}\right)_{i j}$. The oscillator algebra is $(a, b=1,2)$

$$
\begin{aligned}
{\left[\left(b_{a}\right)_{i j},\left(\bar{b}_{b}\right)_{k l}\right] } & =\delta_{a b} \delta_{i l} \delta_{j k} & {\left[\left(b_{a}\right)_{i j},\left(b_{b}\right)_{k l}\right] } & =\left[\left(\bar{b}_{a}\right)_{i j},\left(\bar{b}_{b}\right)_{k l}\right]=0 \\
\left\{\left(f_{a}\right)_{i j},\left(\bar{f}_{b}\right)_{k l}\right\} & =\delta_{a b} \delta_{i l} \delta_{j k} & \left\{\left(f_{a}\right)_{i j},\left(f_{b}\right)_{k l}\right\} & =\left\{\left(\bar{f}_{a}\right)_{i j},\left(\bar{f}_{b}\right)_{k l}\right\}=0
\end{aligned}
$$

The vacuum of Fock space $|0\rangle$ obeys $\left(b_{a}\right)_{i j}|0\rangle=0=\left(f_{a}\right)_{i j}|0\rangle$ for $i, j=1,2, \cdots, p$. The Gauss graph operators are now represented as states in Fock space, as follows

$$
O_{R, r}(\vec{\sigma}) \longleftrightarrow \prod_{\hat{A}=1}^{4} \prod_{i, j=1}^{p}\left(\bar{a}_{\hat{A}}\right)_{i j}^{\left(N_{\hat{A}}\right)_{i \rightarrow j}}|0\rangle
$$

Out next task is to represent the dilatation operator $D_{\hat{A} \hat{B}}$ in the Gauss graph basis. The product of delta functions appearing in (4.34) is not normalized. We will trade it for a delta function normalized to 1

$$
\prod_{\hat{C} \neq \hat{A}, \hat{B}} \delta_{\left(\sigma_{\hat{C}}\right)_{1}\left(\sigma_{\hat{C}}\right)_{2}} \delta\left(\sigma_{1}^{-1} h_{1} \sigma_{2} h_{2}\right)=\left|O_{R, r_{1}}\left(\vec{\sigma}_{1}\right)\right|^{2} \delta_{\left[\vec{\sigma}_{1}\right]\left[\vec{\sigma}_{2}\right]}
$$

where $\delta_{\left[\vec{\sigma}_{1}\right]\left[\vec{\sigma}_{2}\right]}=1$ if permutations $\vec{\sigma}_{1}$ and $\vec{\sigma}_{2}$ belong to the same class of the cosets (2.14), and the delta function vanishes if they are not in the same class. The matrix elements $\left(M_{\hat{A} \hat{B}}\right)_{R, r_{1}, \vec{\sigma}_{1} T, t_{1}, \vec{\sigma}_{2}}$ are only non-zero if we can choose coset representatives such that $\sigma_{1}$ and $\sigma_{2}$ describe the same element of $S_{N_{\hat{A}}} \times S_{N_{\hat{B}}}$. This reflects the fact that the graphs described by $\sigma_{1}$ and $\sigma_{2}$ differ only in the number of edges with both ends attached to the same node, but not in the number of edges between distinct nodes. In this case the matrix 
element in (4.34) simplifies to

$$
\begin{aligned}
\left(M_{\hat{A} \hat{B}}\right)_{R, r_{1}, \vec{\sigma}_{1} T, t_{1}, \vec{\sigma}_{2}}= & \sum_{R^{\prime}} \sqrt{\frac{\left|O_{R, r_{1}}\left(\sigma_{1}\right)\right|^{2}}{\left|O_{T, t_{1}}\left(\sigma_{2}\right)\right|^{2}}} \delta_{r_{1} t_{1}} \delta_{R_{i}^{\prime} T_{k}^{\prime}} \delta_{\left[\vec{\sigma}_{1}\right]\left[\vec{\sigma}_{2}\right]} \sqrt{\frac{\left(N+l_{R_{i}}\right)\left(N+l_{T_{k}}\right)}{l_{R_{i}} l_{T_{k}}}} \\
& \times\left[2 \delta_{i k}\left(N_{\hat{A}}\right)_{i}\left(N_{\hat{B}}\right)_{i}-\left(\left(N_{\hat{A}}\right)_{k i}\left(N_{\hat{B}}\right)_{k k}+\left(N_{\hat{A}}\right)_{k k}\left(N_{\hat{B}}\right)_{i k}\right)\right]
\end{aligned}
$$

Using the oscillators introduced above, we can write number operators whose eigenvalues count the edges in the graph. We will use a hat when we want to describe a number operator which acts on states and no hat when we want to refer to the integer number of edges of a particular graph. For example

$$
\left(\hat{N}_{\hat{A}}\right)_{i i}=\left(\bar{a}_{\hat{A}}\right)_{i i}\left(a_{\hat{A}}\right)_{i i} \quad\left(\hat{N}_{\hat{A}}\right)_{i \rightarrow k}=\left(\bar{a}_{\hat{A}}\right)_{k i}\left(a_{\hat{A}}\right)_{i k}
$$

where there is no sum on $i, k$ in the last formula above, and

$$
\begin{aligned}
\left(N_{\hat{A}}\right)_{i} & =\sum_{k \neq i}\left(\hat{N}_{\hat{A}}\right)_{i \rightarrow k}+\left(\bar{a}_{\hat{A}}\right)_{i i}\left(a_{\hat{A}}\right)_{i i}=\sum_{k}\left(\bar{a}_{\hat{A}}\right)_{k i}\left(a_{\hat{A}}\right)_{i k} \\
& =\sum_{k \neq i}\left(\hat{N}_{\hat{A}}\right)_{k \rightarrow i}+\left(\bar{a}_{\hat{A}}\right)_{i i}\left(a_{\hat{A}}\right)_{i i}=\sum_{k \neq i}\left(\bar{a}_{\hat{A}}\right)_{i k}\left(a_{\hat{A}}\right)_{k i}
\end{aligned}
$$

We can then write the piece of the Hamiltonian of the lattice model we are considering as

$$
\begin{array}{r}
H_{\hat{A} \hat{B}}=\sum_{i, j=1}^{p} \sqrt{\frac{\left(N+l_{R_{i}}\right)\left(N+l_{R_{j}}\right)}{l_{R_{i}} l_{R_{j}}}}\left(-\left(\hat{N}_{\hat{B}}\right)_{j i}\left(\bar{a}_{\hat{A}}\right)_{j j}\left(a_{\hat{A}}\right)_{i i}-\left(\hat{N}_{\hat{A}}\right)_{j i}\left(\bar{a}_{\hat{B}}\right)_{j j}\left(a_{\hat{B}}\right)_{i i}\right. \\
\left.\quad+2 \delta_{i j}\left(\sum_{l \neq i}\left(\hat{N}_{\hat{A}}\right)_{i \rightarrow l}+\left(\bar{a}_{\hat{A}}\right)_{i i}\left(a_{\hat{A}}\right)_{i i}\right)\left(\sum_{l \neq i}\left(\hat{N}_{\hat{B}}\right)_{i \rightarrow l}+\left(\bar{a}_{\hat{B}}\right)_{i i}\left(a_{\hat{B}}\right)_{i i}\right)\right)
\end{array}
$$

The complete Hamiltonian is obtained by summing over $A, B$. Matrix elements of (5.7) computed using the Fock space states are in exact agreement with matrix elements of the one loop dilatation operator, computed in the Gauss graph basis. The mixing matrix in (5.4) is a matrix element so that the normalization of states used to compute the matrix element are reflected in its value. The formula (5.7) is an operator acting in a Fock space and hence is independent of state normalizations. The factor $\sqrt{\frac{\left|O_{R, r_{1}}\left(\sigma_{1}\right)\right|^{2}}{\left|O_{T, t_{1}}\left(\sigma_{2}\right)\right|^{2}}}$ present in $(5.4)$ but absent in (5.7) is due to the normalization of states - see equation (4.36). The Hamiltonian (5.7) leads to a mixing between operators that have different $R$ and $T$ labels, induced by the first two terms appearing in (5.7). Thus, our final result for the Hamiltonian of the lattice model, arising from the one loop dilation operator, is

$$
\begin{aligned}
H= & -\frac{2 g_{\mathrm{YM}}^{2}}{(4 \pi)^{2}} \sum_{\hat{A}=1}^{4} \sum_{i>j=1}^{p}\left(\hat{N}_{\hat{A}}\right)_{i j} \Delta_{i j} \\
& -\frac{2 g_{\mathrm{YM}}^{2}}{(4 \pi)^{2}} \sum_{\hat{A}=1}^{3} \sum_{\hat{B}=1+\hat{A}}^{4} \sum_{i, j=1}^{p} \sqrt{\frac{\left(N+l_{R_{i}}\right)\left(N+l_{R_{j}}\right)}{l_{R_{i}} l_{R_{j}}}}\left(-\left(\hat{N}_{\hat{B}}\right)_{j i}\left(\bar{a}_{\hat{A}}\right)_{j j}\left(a_{\hat{A}}\right)_{i i}-\left(\hat{N}_{\hat{A}}\right)_{j i}\left(\bar{a}_{\hat{B}}\right)_{j j}\left(a_{\hat{B}}\right)_{i i}\right. \\
& \left.+2 \delta_{i j}\left(\sum_{l \neq i}\left(\hat{N}_{\hat{A}}\right)_{i \rightarrow l}+\left(\bar{a}_{\hat{A}}\right)_{i i}\left(a_{\hat{A}}\right)_{i i}\right)\left(\sum_{l \neq i}\left(\hat{N}_{\hat{B}}\right)_{i \rightarrow l}+\left(\bar{a}_{\hat{B}}\right)_{i i}\left(a_{\hat{B}}\right)_{i i}\right)\right)
\end{aligned}
$$




\section{$6 \quad$ Emergent Yang-Mills theory}

The operators we study are labeled by Young diagrams that have $p$ long rows. They are holographically dual to a system of $p$ dual giant gravitons that have expanded to $S^{3} \subset A d S_{5}$. A natural guess is that the dynamics described by the Hamiltonian we have derived arises from the worldvolume dynamics of a system of $p$ giant gravitons. In this section we will confirm this expectation.

This worldvolume theory of the giant graviton branes comes from the dynamics of their open string excitations, so we expect the world volume dynamics is a super YangMills theory. Since the space defined by the brane's world volume is not the space on which the original gauge theory is defined, we will refer to this as an emergent gauge theory [13]. We can say a few things about precisely what theory we expect:

1. Since there are $p$ giant graviton branes we expect a $\mathrm{U}(p)$ gauge theory. Each brane corresponds to a row in the Young diagram, and therefore, to a node in the Gauss graph. The edges which stretch between (not necessarily distinct) nodes will be identified with the open string excitations. Thus, if we label the nodes in the graph with an integer $i=1,2, \cdots, p$, we naturally label the end points of the edges by allowing them to inherit the label of the node. These labels for the end points of the edges are the Chan-Paton indices of the open strings.

2. Before adding any excitations, the operators are constructed from a single field $\phi_{1}$ and are 1/2 BPS. The brane moves in AdS spacetime with metric

$$
d s^{2}=R^{2}\left(-\cosh ^{2} \rho d t^{2}+d \rho^{2}+\sinh ^{2} \rho d \Omega_{3}^{2}\right)
$$

The $\rho$ at which the giant is located is specified by

$$
\cosh \rho=\sqrt{1+\frac{l_{R}}{N}} \quad \sinh \rho=\sqrt{\frac{l_{R}}{N}}
$$

with $l_{R}$ the length of the row in $R$ corresponding to the giant graviton brane. We are in the displaced corners approximation, which implies that the row lengths of our operators are unequal and hence the $p$-branes are separated in spacetime. Consequently we are studying the gauge theory on its Coulomb branch. In the low energy limit the dynamics is described by a $\mathrm{U}(1)^{p}$ gauge theory. This nicely matches what we find: our dynamical fields are the closed loops formed by edges located at a given node - these are the only edges that are changed by the action of the dilatation operator. The open strings corresponding to these dynamical edges have both end points labeled by the same gauge group index, so they belong to the diagonal $\mathrm{U}(1)^{p}$. There is one $\mathrm{U}(1)$ for each node. Notice that $g_{\mathrm{YM}}^{2}$ of the emergent theory is equal to the $\mathrm{AdS}_{5} \times \mathrm{S}^{5}$ string coupling which is itself equal to the original coupling $g_{\mathrm{YM}}^{2}$ of the $\mathcal{N}=4$ super Yang-Mills theory we study. Since we are studying weak coupling in the original Yang-Mills theory, we are at weak coupling in the emergent gauge theory.

3. We have not studied the complete $\mathcal{N}=4$ super Yang-Mills theory, since we have truncated to the $\mathrm{su}(2 \mid 3)$ sector. Consequently, we will only recover part of the expected 
$\mathrm{U}(1)^{p}$ gauge theory. The bosonic part of the symmetry of $\mathcal{N}=4$ super Yang-Mills theory is $\mathrm{SO}(2,4) \times \mathrm{SO}(6)$. The $\mathrm{SO}(4)$ that acts as the isometry of the brane world volume is a subgroup of $\mathrm{SO}(2,4)$; this $\mathrm{SO}(4)$ is a spacetime symmetry of the world volume theory. The $\mathrm{SO}(4)$ which rotates the real components of the $\phi_{2}, \phi_{3}$ fields is a subgroup of $\mathrm{SO}(6)$; this $\mathrm{SO}(4)$ is a global symmetry of the world volume theory. Consequently the excitations constructed from the $\phi_{2}, \phi_{3}$ fields are scalar fields of the emergent gauge theory. $\psi_{1}$ and $\psi_{2}$ are their super partners. Finally, our truncation to the $\mathrm{su}(2 \mid 3)$ sector retains only fields invariant under the $\mathrm{SO}(4) \subset \mathrm{SO}(4,2)$, so that we should expect to reproduce the s-wave sector of the emergent gauge theory.

Thus, we should compare our emergent theory to the low energy limit of a $\mathrm{U}(p)$ gauge theory on its Coulomb branch. We expect to reproduce the $s$-wave sector of the dynamics of the adjoint scalars and their super partners.

With these comments in mind, we now consider the action for the adjoint scalars of a $\mathrm{U}(p)$ gauge theory, defined on an $\mathrm{S}^{3}$, which has the form

$$
\begin{gathered}
S=\frac{1}{g_{\mathrm{YM}}^{2}} \int_{\mathbb{R} \times S^{3}}\left[\operatorname{Tr}\left(\partial_{\mu} X \partial^{\mu} X^{\dagger}+\partial_{\mu} Y \partial^{\mu} Y^{\dagger}-\frac{1}{R^{2}}\left(X X^{\dagger}+Y Y^{\dagger}\right)-[X, Y]\left[Y^{\dagger}, X^{\dagger}\right]\right)\right. \\
\left.-\sum_{i \neq j} m_{i j}^{2}\left(X_{i j} X_{j i}^{\dagger}+Y_{i j} Y_{j i}^{\dagger}\right)\right] d t R^{3} d \Omega_{3}
\end{gathered}
$$

$R$ is the radius of the $\mathrm{S}^{3}$ on which the theory is defined and the $1 / R^{2}$ terms are required for conformal invariance, as usual. The off diagonal matrix elements of adjoint scalars $X, Y$ will have masses $m_{i j}$ proportional to the distances separating the branes between which they stretch. Truncating to the $s$-wave sector gives the matrix quantum mechanics

$$
\begin{aligned}
S=\frac{R^{3} \Omega_{3}}{g_{\mathrm{YM}}^{2}} \int_{\mathbb{R}} & {\left[\operatorname{Tr}\left(\dot{X} \dot{X}^{\dagger}+\dot{Y} \dot{Y}^{\dagger}-\frac{1}{R^{2}}\left(X X^{\dagger}+Y Y^{\dagger}\right)-[X, Y]\left[Y^{\dagger}, X^{\dagger}\right]\right)\right.} \\
& \left.-\sum_{i \neq j} m_{i j}^{2}\left(X_{i j} X_{j i}^{\dagger}+Y_{i j} Y_{j i}^{\dagger}\right) d t\right]
\end{aligned}
$$

The eigenvalues of the one loop dilatation operator give the spectrum of anomalous dimensions. Identifying the classical contribution to the dimension with the free part of the emergent gauge theory, the dynamics obtained from the one loop dilatation operator should match the interaction Hamiltonian, given by

$$
H_{\mathrm{int}}=\frac{R^{3} \Omega_{3}}{g_{\mathrm{YM}}^{2}}\left[\sum_{i \neq j} m_{i j}^{2}\left(X_{i j} X_{j i}^{\dagger}+Y_{i j} Y_{j i}^{\dagger}\right)+\operatorname{Tr}\left([X, Y]\left[Y^{\dagger}, X^{\dagger}\right]\right)\right]
$$

The operators studied in earlier sections are constructed using $\phi_{2}, \phi_{3}$ and not $\phi_{2}^{\dagger}, \phi_{3}^{\dagger}$. This 
truncation must also be accounted for. This is achieved by truncating the mode expansions

$$
\begin{aligned}
X & =\frac{1}{\sqrt{2}}(\tilde{a}+\bar{a}) & X^{\dagger} & =\frac{1}{\sqrt{2}}(a+\overline{\tilde{a}}) \\
Y & =\frac{1}{\sqrt{2}}(\tilde{b}+\bar{b}) & Y^{\dagger} & =\frac{1}{\sqrt{2}}(b+\overline{\tilde{b}})
\end{aligned}
$$

The truncation sets all tilded oscillators to zero, i.e. we replace $X \rightarrow \bar{a}, X^{\dagger} \rightarrow a, Y \rightarrow \bar{b}$ and $Y^{\dagger} \rightarrow b$. The interaction Hamiltonian becomes (we are assuming normal ordering for $H_{\text {int }}$ )

$$
H_{\mathrm{int}}=\frac{R^{3} \Omega_{3}}{g_{\mathrm{YM}}^{2}}\left[\sum_{i \neq j} m_{i j}^{2}\left(\bar{a}_{i j} a_{j i}+\bar{b}_{i j} b_{j i}\right)+\operatorname{Tr}([\bar{b}, \bar{a}][a, b])\right]
$$

The first term in the interaction Hamiltonian matches the terms in the dilatation operator with action given in (4.10). To see the equality, note that at large $N$ we are justified in ignoring the difference between $O_{R_{i j}^{+},\left(r_{i j}^{+}, s\right) \mu_{1} \mu_{2}}$ and $O_{R,(r, s) \mu_{1} \mu_{2}}$, which amounts to ignoring the effects of back reaction, due to the open string excitations, on the size of the giants. Once the back reaction is ignored, the action quoted in (4.10) simplifies nicely. For example ${ }^{6}$

$$
D_{\phi_{1} \phi_{2}}|\sigma\rangle=-\sum_{i, j=1}^{p}\left(\sqrt{N+l_{R_{i}}}-\sqrt{N+l_{R_{j}}}\right)^{2} \bar{a}_{i j} a_{i j}|\sigma\rangle
$$

How should we interpret this answer? Our giant gravitons are constructed mainly from $\phi_{1}$ fields, with a small number of excitations. Consequently, they are small deformations of $\frac{1}{2}$ BPS operators. A very natural set of coordinates for the study of $\frac{1}{2}$ BPS geometries in the dual gravitational theory was given by Lin, Lunin and Maldacena in [42]. The geometry is written in terms of two three spheres, time $t$ and three more spacial coordinates $y, x_{1}, x_{2}$. In terms of these coordinates, the $\mathrm{AdS}_{5} \times \mathrm{S}^{5}$ geometry corresponds to a circular droplet boundary condition on the $y=0$ plane, parameterized by the $\left(x_{1}, x_{2}\right)$ coordinates (see section 2.3 of [42]). Introduce radial coordinates $(r, \phi)$ on this plane. The $r$ and $y$ coordinates are related to $\rho$ (the radial variable of $\mathrm{AdS}_{5}$ in global coordinates) and $\theta$ (one of the angles of the $\left.\mathrm{S}^{5}\right)$ by $y=r_{0} \sinh \rho \sin \theta$ and $r=r_{0} \cosh \rho \cos \theta$, where $r_{0}=R_{\mathrm{AdS}_{5}}^{2}=R_{\mathrm{S}^{5}}^{2}$. The dual giant gravitons corresponding to a row of length $l_{R}$ is located at

$$
\theta=0 \quad \cosh \rho=\sqrt{1+\frac{l_{R}}{N}}
$$

so that

$$
y=0 \quad r=\sqrt{1+\frac{l_{R}}{N}}
$$

From the $\mathrm{AdS}_{5} \times \mathrm{S}^{5}$ geometry written in LLM coordinates, we find that the metric on the LLM plane at $y=0$ is given by $d s^{2}=\left(d x_{1}\right)^{2}+\left(d x_{2}\right)^{2}=d r^{2}+r^{2} d \phi^{2}$. Thus, the coefficient in (6.8) is square of the proper distance between the branes corresponding to rows $i$ and

\footnotetext{
${ }^{6}$ There is a similar result for all $D_{\phi_{1} \hat{A}}$ terms.
} 
$j$ of $R$. This proves that (4.10) reproduces the first term in (6.7) after identifying $a_{i j}, \bar{a}_{i j}$ with $\left(b_{1}\right)_{i j},\left(\bar{b}_{1}\right)_{i j}$. Notice further that the squared masses are indeed proportional to the square of distances between branes. In the same way, the oscillators $b_{i j}, \bar{b}_{i j}$ will produce the required mass terms for $\left(b_{2}\right)_{i j},\left(\bar{b}_{2}\right)_{i j}$.

Now consider the commutator squared term

$$
\operatorname{Tr}([\bar{b}, \bar{a}][a, b])=\operatorname{Tr}(\bar{b} \bar{a} a b+\bar{a} \bar{b} b a-\bar{a} \bar{b} a b-\bar{b} \bar{a} b a)
$$

We need to perform a truncation to obtain the low energy theory. The truncation will freeze the dynamics of the massive modes. This is most simply illustrated with a specific example: consider the term $\operatorname{Tr}(\bar{b} \bar{a} a b)=\bar{b}_{i j} \bar{a}_{j k} a_{k l} b_{l i}$. Borrowing the language of the Gauss graph to make the discussion transparent, this term destroys a $b$ edge stretching from $i$ to $l$ and creates a $b$ edge stretching from $i$ to $j$. To freeze the edges stretched between nodes we should keep only the terms with $j=l$. Truncating to achieve this we find

$$
\begin{aligned}
\operatorname{Tr}(\bar{b} \bar{a} a b) & =\bar{b}_{i j} \bar{a}_{j k} a_{k l} b_{l i} \\
& \rightarrow \bar{b}_{i j} \bar{a}_{j k} a_{k j} b_{j i}=\bar{b}_{i j} b_{j i} \bar{a}_{j k} a_{k j} \\
& =\sum_{j}\left(\sum_{i \neq j}\left(\hat{n}_{3}\right)_{j \rightarrow i}+\left(\hat{n}_{3}\right)_{j j}\right)\left(\sum_{k \neq j}\left(\hat{n}_{2}\right)_{j \rightarrow k}+\left(\hat{n}_{2}\right)_{j j}\right)
\end{aligned}
$$

This truncation can be viewed as a Born-Oppenheimer approximation, in which we fix the edges stretched between nodes and solve the dynamics of the light edges. This will be a good approximation as long as we don't excite the light edges to an energy comparable to that of the stretched edges. Truncating the remaining terms in the commutator squared, we find

$$
\begin{aligned}
\operatorname{Tr}(\bar{a} \bar{b} b a) & \rightarrow\left(\sum_{i \neq j}\left(\hat{n}_{2}\right)_{j \rightarrow i}+\left(\hat{n}_{2}\right)_{j j}\right)\left(\sum_{k \neq j}\left(\hat{n}_{3}\right)_{j \rightarrow k}+\left(\hat{n}_{3}\right)_{j j}\right) \\
-\operatorname{Tr}(\bar{a} \bar{b} a b) & \rightarrow-\left(\hat{n}_{2}\right)_{i j} \bar{b}_{j j} b_{i i}-\left(\hat{n}_{3}\right)_{k j} \bar{a}_{j j} a_{k k}+\left(\hat{n}_{2}\right)_{i i}\left(\hat{n}_{3}\right)_{i i} \\
-\operatorname{Tr}(\bar{b} \bar{a} b a) & \rightarrow-\left(\hat{n}_{3}\right)_{j i} \bar{a}_{j j} a_{i i}-\left(\hat{n}_{2}\right)_{k j} \bar{b}_{j j} b_{k k}+\left(\hat{n}_{2}\right)_{i i}\left(\hat{n}_{3}\right)_{i i}
\end{aligned}
$$

Summing the four terms above we reproduce (5.7) in complete detail, up to the overall factor. The overall factor given by

$$
\propto-g_{\mathrm{YM}}^{2} \sqrt{\frac{\left(N+l_{R_{i}}\right)\left(N+l_{R_{j}}\right)}{l_{R_{i}} l_{R_{j}}}}
$$

is perfectly explained as the field redefinition needed to match the dual giant graviton solution to a BPS classical solution of super Yang-Mills theory on $\mathbb{R} \times \mathrm{S}^{3}$ [7]. See appendix A for a detailed discussion.

\section{$7 \quad$ Mixing with closed string states}

Consider the dilatation operator we have derived in (5.8). The terms on the first line of (5.8) are of order $\lambda=g_{\mathrm{YM}}^{2} N$, whilst those on the second and third lines are of order $\frac{\lambda}{N}$, 
so that they are $O\left(N^{-1}\right)$ corrections. Are there additional $O\left(N^{-1}\right)$ terms that have not been included in our computation? In this section we will deal with this issue carefully.

One source of $O\left(N^{-1}\right)$ corrections is from mixing with operators that have some short rows or columns. The operators we have considered have $p$ long rows or columns. For example, operators with $p=3$ long rows, dual to a state of $p=3$ dual giant gravitons would have a Young diagram $R$ given by

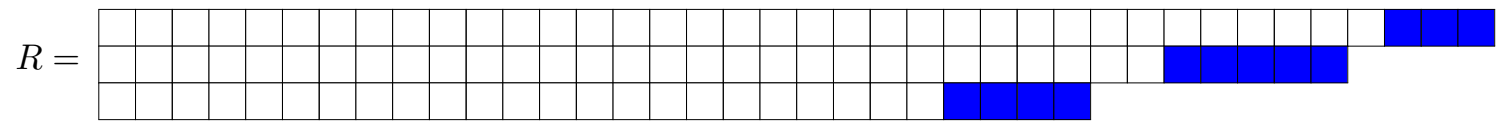

The solid boxes are excitations of the dual giant gravitons. The number of hollow boxes in each row is the number of $Z$ fields in the corresponding giant graviton, which is the angular momentum of the giant. The long rows are gravitons with angular momentum $\sim N$, which is why they have expanded to some macroscopic size. This is the usual Myers effect [40]. There are also operators that have a small number of extra columns, but with the same total number of boxes

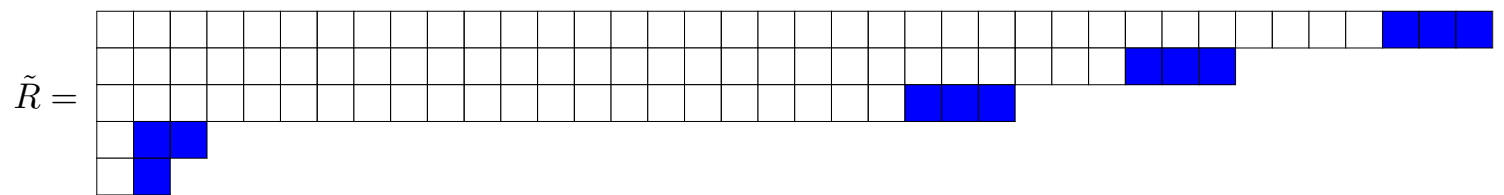

Mixing with these operators is suppressed in the large $N$ limit, but once $1 / N$ corrections are included, mixing is allowed. The short rows are gravitons with a small $\sim O(1)$ momentum. With such a small angular momentum, these gravitons are simply point like gravitons and other closed string states. Thus, the long rows correspond to dual giant graviton branes and their open string excitations, while the short rows are closed string excitations, as has been explained in [9]. Physically this makes perfect sense: the states dual to a system of $p$ giant gravitons will include both open string excitations (of the giant graviton branes) and closed string excitations (of the spacetime). The total Hilbert space of string excitations is of the form

$$
\mathcal{H}=\mathcal{H}_{\text {open }} \otimes \mathcal{H}_{\text {closed }}
$$

Thus, there are indeed $1 / N$ corrections to the dilation operator that we did not include in our analysis. However, these terms correspond to making a transition from an open to a closed string and they mix open string states with (closed string) states that do not belong to the open string Hilbert space. These terms would be responsible for closed string absorption and emission from the D-branes and their effect has been studied in $[13,14]$. To perturb the energy eigenvalues (and hence the anomalous dimensions we compute) these terms first need to produce a transition from an open to a closed string state, and then they need to produce a second transition back into the open string Hilbert space. Thus these terms produce a $\lambda^{2} / N^{2}$ correction to the eigenvalue, ${ }^{7}$ which is exactly of the correct size to

${ }^{7} \mathrm{~A}$ simple example maybe useful to illustrate our point: the matrix

$$
\left(\begin{array}{cc}
a & \frac{1}{N} \\
\frac{1}{N} & b
\end{array}\right)
$$


be interpreted as a closed string correction. This can be dropped since we are computing the order $\frac{\lambda}{N}$ terms in the spectrum.

There is another potential source of corrections: consider (6.8). The term on the right hand side of $(6.8)$ is of order $N$. In general we should expect $O(1)$ corrections to modify this to

$$
D_{\phi_{1} \phi_{2}}|\sigma\rangle=-\sum_{i, j=1}^{p}\left(\sqrt{N+l_{R_{i}}}-\sqrt{N+l_{R_{j}}}\right)^{2} \bar{a}_{i j} a_{i j}|\sigma\rangle+O(1)
$$

These $O(1)$ corrections will be of the same order of magnitude as the order $\frac{\lambda}{N}$ terms we have computed. How do they affect the spectrum? First order corrections to the state will again translate into second order corrections to the spectrum, by the argument just reviewed, so that we need not consider these corrections to the order that we work. However, $N^{-1}$ corrections to the eigenvalue in (6.8) will most certainly change our result. In the papers $[21,41]$ this eigenvalue problem was studied in the $\mathrm{SU}(2)$ sector numerically. The exact matrix elements of the diltation operator were computed without invoking any of the simplifications of large $N$. Mixing with states of short columns was put to zero. Remarkably, as shown in $[22,31]$, after dropping the mixing with closed string states the exact spectrum of the resulting problem matches the spectrum computed after using the displaced corners approximation! Thus, there are no $N^{-1}$ corrections to eigenvalues once mixing with closed string states are dropped. We assume that these conclusions hold when one has more than 3 rows. $^{8}$ With this assumption there are no $N^{-1}$ corrections to the eigenvalue in (6.8). To further support this conclusion, we have explicitely studied the $1 / N$ correction to the dilatation operator in appendix B.

When do we expect the Gauss graph basis to be meaningful and when do we expect it to break down? As a warm up, consider first the planar limit. We are considering single trace operators with a bare dimension that is at most $\sim \sqrt{N}$. These operators provide a basis for the closed string Hilbert space $\mathcal{H}_{\text {closed }}$. In the planar limit, higher genus corrections are supressed by $N^{-2}$, so large $N$ corresponds to weak string coupling. As the string coupling increases ( $N$ gets smaller) interactions become important and there is mixing between string states with different numbers of strings. This corresponds to a mixing between different trace structures. A description in terms of weakly interacting single string states is no longer appropriate, and the description in terms of a trace basis becomes less and less meaningful. Lets now consider the Gauss graph basis. The Gauss graphs provide a basis for the open string Hilbert space $\mathcal{H}_{\text {open }}$. Nodes of the graph correspond to branes and edges to open strings. In the displaced corners approximation that we use in this paper, the edges stretched between distinct nodes have a much larger energy (of size $\lambda$ ) than the edges with has eigenvalues $\lambda=\left\{b-\frac{1}{(a-b) N^{2}}+O\left(N^{-4}\right), a+\frac{1}{(a-b) N^{2}}+O\left(N^{-4}\right)\right\}$ so that the $N^{-1}$ terms off the diagonal produce an $N^{-2}$ correction to the eigenvalue.

${ }^{8} \mathrm{~A}$ single row is not generic - all restricted Schur polynomials constructed with $R$ a single row or a single column are annihilated by the one loop dilatation operator. As soon as there are two (or more) rows or columns the one loop dilatation operator has a non-zero action and there are non-zero anomalous dimensions. There are no new features that arise when going from two rows (or columns) to many rows (or columns). 
both ends attached to a single node (which has an energy of order $\frac{\lambda}{N}$ ). This corresponds to the Coulomb branch of the gauge theory and the low energy dynamics will match a $\mathrm{U}(1)^{p}$ gauge theory. When the corners of the Young diagram $R$ approach each other, the open string description continues to be valid, but now the energies of edges stretched between different nodes and of edges with endpoints on the same node are comparable. In this situation the approximations used in computing the action of the dilatation operator are no longer valid. We expect a correct computation to show that all edges become dynamical with the dynamics matching a $\mathrm{U}(p)$ gauge theory at low energy. The Gauss graph basis itself should continue to be a useful basis as long as the semiclassical open string description is appropriate. In the intermeddiate regime where row or column lengths are much larger than $\sim \sqrt{N}$ but much less than $N$ the Gauss graph basis does break down and we do not have a good guess for what replaces it.

\section{Conclusions and outlook}

In this article we have studied the operator mixing problem for operators dual to systems of excited dual giant graviton branes. The description we have constructed has a number of interesting features. The mixing problem is simply described using a basis labeled by a pair of Young diagrams $R$ and $r_{1}$ and a graph $\vec{\sigma}$. The Young diagram $r_{1}$ organizes the $\phi_{1}$ fields in the operator. In the dual holographic theory, each row corresponds to a dual giant graviton. The length of the rows of $r_{1}$ is equal to the number of $\phi_{1}$ fields used to construct the giant and this gives the momentum and hence the size of the (square of the) dual giant graviton. The Young diagram $R$ plays a very similar role, except that it includes the excitations in the description. The graph specifies the state of the excitations. Nodes of the graph correspond to the giant gravitons, while the excitations are represented as edges with end points attached to the nodes. The matrix elements of the dilatation operator are written in terms of the number of edges appearing in the graph. Interpreting the edges as an occupation number representation, we have mapped each Gauss graph operator into a Fock space state and we have mapped the dilatation operator into a Hamiltonian acting on this Fock space. We have identified this description with the Fock space of the emergent gauge theory, realized as the giant world volume theory.

One obvious extension of our results would be to relax the truncation to the $\mathrm{su}(2 \mid 3)$ sector. By including fields that are not invariant under the $\mathrm{SO}(4)$ rotating the world volume we go beyond the $s$-wave sector. This would start to reconstruct the spatial dependence of the world volume theory and constructing this aspect of the world volume theory maybe a useful toy model for the emergence of spacetime in general. Including the gauge fields for example, would be straight forward given the results already obtained in [43]. This would already be a fascinating and non-trivial extension. Including further types of excitations would increase the number of Young diagrams labels on the restricted Schur polynomials, as well as increasing the number of species of edges in the Gauss graphs.

As the number of giant gravitons grows one enters into the regime where back reaction can't be ignored. In the description developed here, increasing the number of giant gravitons implies the number of nodes in the graph grows. When the number of nodes 
becomes of order $N$, back reaction becomes important. In the 1/2-BPS sector for example, states of $N$ giant gravitons back react to produce the LLM geometries [42]. In this regime the operators we study correspond to new spacetime geometries and it is interesting to ask if signatures of the gravitational dynamics are visible. The out-of-time-order correlator (OTOC) provides a signal of a possible gravity dual. Holographic computations which consider shock waves in black hole geometries, has led to a bound on the quantum Lyapunov exponent, evaluated using thermal OTOCs [44]. The black hole geometries saturate the bound, with the maximum value attributed to the red shift near the event horizon of the black hole. To compute the thermal average we must average over all of the states in the Fock space. Since the numbers $\left(N_{\hat{A}}\right)_{i \rightarrow l}$ with $i \neq l$ label the state, the sum over states can be written as a sum over these integers. These same numbers appear as parameters in the Hamiltonian so that we are naturally lead to study a model for particles hopping on a lattice with of the order of $N$ sites, with quenched disorder and, for the generic state, hopping can happen between any two sites in the lattice i.e. all sites are connected. These look a lot like the SYK models $[45,46]$ which are known to saturate the chaos bound [47], suggesting that the computation of the OTOC for the lattice model developed here would be interesting. Of course, the regime in which we expect to get a weakly curved gravity description is the limit of large 't Hooft coupling and our dynamics is only one loop. Nevertheless, the fact that to understand large $N$ but non-planar limits of $\mathcal{N}=4$ super Yang-Mills theory naturally leads to models with quenched disorder and all-to-all interactions between the different sites, is interesting.

Another direction worth pursuing concerns the global symmetry of the model. The dynamics of magnons in the planar limit is tightly constrained by the $\mathrm{su}(2 \mid 2)$ symmetry of the model in an interesting way [48]. The magnon "polarizations" fill out the fundamental representation of a centrally extended $\mathrm{su}(2 \mid 2)$ algebra, which enlarges the original algebra by two central charges $P$ and $K$. These two additional central charges are related to gauge transformations which act non-trivially on individual fields. By requiring that they annihilate the total state, one returns to the original global su(2|2) symmetry. This construction has a number of far reaching consequences. First, it proves that the total anomalous dimension is a sum of contributions, one from each magnon. Second, the kinematics of the global symmetry completely fixes the $S$-matrix, up to an overall phase. The operators we study in this article enjoy the same global symmetry. Is there a similar analysis to be developed for the operators dual to excited giant graviton branes? This question was first explored in [29]. Recall that the lightest string modes of a string stretching between two flat parallel and separated D-branes fill out a massive short representation of the unbroken supersymmetry of the D-brane system. These representations require a central charge extension of the unbroken supersymmetry algebra. The additional central charge has a physical interpretation as an electric charge carried by the open string end-points so that closed string states are not charged. An important conclusion of [29] is that this open string central charge is a limit of the central charge extension of $[48,49]$. The question was reconsidered in [35] using the language of the Gauss graph operators. In the Gauss graph language, the magnons are the edges in the Gauss graph. The conclusion of [35] is that edges stretched between nodes of the Gauss graph do carry the central charge, 
while edges living at a node are not charged. The central extension again generates gauge transformations so that it again vanishes when acting on physical states which are gauge invariant. In the double coset setting the constraint enforced by the Gauss Law (discussed in section 2.2) ensures that the central extension vanishes. In the emergent dynamics that we have constructed in this article, edges with both ends attached to a single node are gauge invariant, which immediately forces the central charge $P$ and $K$ to vanish for these edges. This prevents us from repeating the analysis of $[48,49]$ to learn about the spectrum of anomalous dimensions and the $S$-matrix of two magnon scattering. It remains an interesting exercise to determine the constraints implied by the global $\mathrm{su}(2 \mid 2)$ symmetry.

\section{Acknowledgments}

We would like to thank David Berenstein, Antal Jevicki and Sanjaye Ramgoolam for penetrating insights that were helpful in completing this project. This work is supported by the Science and Technology Program of Guangzhou (No. 2019050001 and No. 2020A1515010388), by a Simons Foundation Grant Award ID 509116 and by the South African Research Chairs initiative of the Department of Science and Technology and the National Research Foundation.

\section{A Field redefinition}

The dual giant graviton solution has been matched to a BPS classical solution of super Yang-Mills theory on $\mathbb{R} \times S^{3}$ [7]. There is a non-trivial field redefinition needed when passing from the field theory to the gravitational description. In this section we will review this field redefinition as it is needed when we compare our emergent dynamics to the expected Yang-Mills theory.

To start, consider a Yang-Mills theory defined on $\mathbb{R} \times \mathrm{S}^{3}$, and denote the radius of the $S^{3}$ by $R$. The Abelian part of the Yang-Mills action for an adjoint scalar, after reducing to the $s$-wave, is

$$
S=\frac{R^{3} \Omega_{3}}{2 g_{\mathrm{YM}}^{2}} \int d t\left(\dot{X} \dot{X}^{\dagger}-\frac{1}{R^{2}} X X^{\dagger}\right)
$$

Reparametrizing the field as

$$
X=\sqrt{\frac{g_{\mathrm{YM}}^{2} N}{R^{2} \Omega_{3}}} \phi
$$

the action becomes

$$
S=\frac{N R}{2} \int d t\left(\dot{\phi} \dot{\phi}^{\dagger}-\frac{\phi \phi^{\dagger}}{R^{2}}\right)
$$

Setting $\phi=\eta e^{i \omega t}$ the classical equations of motion are obeyed when

$$
\eta=\sqrt{\frac{L}{N}}
$$


with $L$ the angular momentum of the dual giant graviton. Further, the energy of this solution is $E=L$. This matches the radius and energy of the dual giant graviton solution obtained using the DBI action [6, 7].

This field redefinition is need for us to compare the emergent lattice dynamics to the gauge theory world volume dynamics of the brane. The field redefinition needed in our study has a number of interesting features. Each row in Young diagram $r_{1}$ corresponds to a dual giant graviton. The number of boxes in the row gives the angular momentum of the row and the square root of this gives the radius of the giant world volume [5], i.e. the $i$ th giant has a radius

$$
R=\sqrt{l_{r_{i}}}=\sqrt{l_{R_{i}}}
$$

where the second equality is true at large $N$ in the displaced corners limit. Next, a number of studies [50-55] have established that when fields that correspond to boxes on a large Young diagram interact, they do so with an effective 't Hooft coupling obtained by replacing $N g_{\mathrm{YM}}^{2} \rightarrow N_{\text {eff }} g_{\mathrm{YM}}^{2}$, with $N_{\text {eff }}$ given by the factor of the box that is interacting. For boxes appearing in the $i$ th row of $r_{1}$ we should replace

$$
N g_{\mathrm{YM}}^{2} \rightarrow\left(N+l_{R_{i}}\right) g_{\mathrm{YM}}^{2}
$$

With these two replacements, the field redefinitions needed in section 6 are ( $a, b$ are oscillators for the $X$ and $Y$ fields, while $b_{1}, b_{2}$ are oscillators for the $\phi_{1}, \phi_{2}$ fields)

$$
a_{i i}=\sqrt{\frac{g_{\mathrm{YM}}^{2}\left(N+l_{R_{i}}\right)}{l_{R_{i}} \Omega_{3}}}\left(b_{1}\right)_{i i} \quad b_{i i}=\sqrt{\frac{g_{\mathrm{YM}}^{2}\left(N+l_{R_{i}}\right)}{l_{R_{i}} \Omega_{3}}}\left(b_{2}\right)_{i i}
$$

as well as the dagger of these equations.

\section{B $\quad N^{-1}$ corrections to matrix elements of the dilatation operator}

In this appendix we study the form of the $1 / N$ corrections to the leading one loop dilatation operator. These corrections when included in (4.10) may produce an $O(1)$ correction to the anomalous dimension, which is then of the same size as the corrections considered in section 5 .

Our starting point is the exact matrix element given in (3.10), when we choose $A=\phi_{1}$. The large $N$ limit is used to simplify the computation of the trace

$$
\begin{aligned}
\operatorname{Tr}_{R \oplus T}\left(\left[\Gamma^{(R)}\right.\right. & \left.\left(\left(1,1_{\phi_{1}}\right)\right) P_{R,(\vec{r} \vec{s}) \vec{\mu} \vec{\nu}} \Gamma^{(R)}\left(\left(1,1_{\phi_{1}}\right)\right), \Gamma^{(R)}\left(\left(1,1_{B}\right)\right)\right] I_{R^{\prime} T^{\prime}} \\
& \left.\times\left[\Gamma^{(T)}\left(\left(1,1_{\phi_{1}}\right)\right) P_{T,(\vec{t} \vec{u}) \vec{\alpha} \vec{\beta}} \Gamma^{(T)}\left(\left(1,1_{\phi_{1}}\right)\right), \Gamma^{(T)}\left(\left(1,1_{B}\right)\right)\right] I_{T^{\prime} R^{\prime}}\right)
\end{aligned}
$$

appearing in $\left(\mathcal{M}_{\phi_{1} B}\right)_{R,(\vec{r} \vec{s}) \vec{\mu} \vec{\nu}, T,(\vec{t} \vec{u}) \vec{\alpha} \vec{\beta}}$. In evaluating the above formula, the permutations $\left(1,1_{\phi_{1}}\right)$ and $\left(1,1_{B}\right)$ are allowed to act on the intertwining maps [22]. The boxes corresponding to the labels $1,1_{\phi_{1}}$ and $1_{B}$ are all boxes situated near the end of a row on the right hand side of the Young diagram. In the displaced corners limit, this permutation simplifies significantly. To see this, it is simplest to use a concrete representation for the matrices of the 
representations $R$ and $T$, known as Young's orthogonal representation. This representation can be described by specifying the action of the group elements on the Young-Yamanouchi basis. We need only specify the "adjacent permutations" which correspond to cycles of the form $(i, i+1)$ because these generate the complete group. Recall that the content of the box in the $i^{\text {th }}$ row and the $j^{\text {th }}$ column is given by $j-i$. Denote the content of the box $l$ by $c_{l}$. Let $\hat{T}$ denote a Young tableau obtained by labeling Young diagram $T$ and let $\hat{T}_{i j}$ denote exactly the same tableau, but with boxes $i$ and $j$ swapped. The rule for the action of the group elements on the Young-Yamanouchi basis is

$$
\Gamma_{T}((i, i+1))|\hat{T}\rangle=\frac{1}{c_{i}-c_{i+1}}|\hat{T}\rangle+\sqrt{1-\frac{1}{\left(c_{i}-c_{i+1}\right)^{2}}}\left|\hat{T}_{i, i+1}\right\rangle
$$

There are two possibilities: either boxes $i$ and $i+1$ belong to the same row, in which case $c_{i}-c_{i+1}=1$ and

$$
\Gamma_{T}((i, i+1))|\hat{T}\rangle=|\hat{T}\rangle
$$

or boxes $i$ and $i+1$ belong to different rows, in which case $c_{i}-c_{i+1}=O(N)$ and at large $N$ we have

$$
\Gamma_{T}((i, i+1))|\hat{T}\rangle=\left|\hat{T}_{i, i+1}\right\rangle+O\left(N^{-1}\right)
$$

It is the correction on the right hand side of the above equation that must be evaluated to determine the $O\left(N^{-1}\right)$ correction to the dilatation operator. Before we discuss the form of the correction there are a few points worth noting. First, notice that in the displaced corners approximation the above action of the symmetric group has a very simple interpretation: if boxes being permuted belong to different rows, they are just swapped. Second, its clear what the implication of this simplified action is for the dilatation operator: the quantities $\Gamma^{(R)}\left(\left(1,1_{\phi_{1}}\right)\right) P_{R,(\vec{r} \vec{s}) \vec{\mu} \vec{\nu}} \Gamma^{(R)}\left(\left(1,1_{\phi_{1}}\right)\right)$ and $\Gamma^{(T)}\left(\left(1,1_{\phi_{1}}\right)\right) P_{T,(\vec{t} \vec{u}) \vec{\alpha} \vec{\beta}} \Gamma^{(T)}\left(\left(1,1_{\phi_{1}}\right)\right)$ are the original intertwining maps, but with the $\phi$ field that is mixing transported to the slot labeled 1. Consequently the permutations $\Gamma^{(R)}\left(\left(1,1_{B}\right)\right)$ and $\Gamma^{(T)}\left(\left(1,1_{B}\right)\right)$ are swapping the boxes that correspond to the $\phi_{1}$ and $B$ fields that are mixing. These two facts together imply that it is possible for a $B$ field to change rows, that is, both or neither of the "endpoints" of a given $Y$ field are transported. This then implies that edges that are attached to one node can detach and reattach to a different node, but edges with ends attached to different nodes are fixed. Of course, because the endpoints of each edge have opposite charge this does not change the Chan-Paton charges of the dual open string state.

Now consider the correction of order $N^{-1}$ appearing in (B.2). This correction implies that when a permutation acts it swaps the endpoints with a matrix element of size $\sim 1$, or leaves them inert with a matrix element of size $\sim N^{-1}$. When considering the actions on the two endpoints, there is a correction to the leading action of the dilatation operator in which we transport only a single endpoint of an edge. This correction will therefore always change the Chan-Paton charges of the state and consequently, it corrects off diagonal elements of the dilatation operator. Importantly, there are no corrections to the diagonal elements of the dilatation operator at the next to leading order in a large $N$ expansion. Consequently, these will induce corrections to the form of the operators of a good scaling dimension, but not to the dimension of the operator. The conclusion is that the possible order 1 correction to the anomalous dimension vanishes. 
Open Access. This article is distributed under the terms of the Creative Commons Attribution License (CC-BY 4.0), which permits any use, distribution and reproduction in any medium, provided the original author(s) and source are credited.

\section{References}

[1] N. Beisert et al., Review of AdS/CFT Integrability: An Overview, Lett. Math. Phys. 99 (2012) 3 [arXiv: 1012.3982] [inSPIRE].

[2] J.M. Maldacena, The Large $N$ limit of superconformal field theories and supergravity, Int. J. Theor. Phys. 38 (1999) 1113 [Adv. Theor. Math. Phys. 2 (1998) 231] [hep-th/9711200] [INSPIRE].

[3] S.S. Gubser, I.R. Klebanov and A.M. Polyakov, Gauge theory correlators from noncritical string theory, Phys. Lett. B 428 (1998) 105 [hep-th/9802109] [INSPIRE].

[4] E. Witten, Anti-de Sitter space and holography, Adv. Theor. Math. Phys. 2 (1998) 253 [hep-th/9802150] [INSPIRE].

[5] J. McGreevy, L. Susskind and N. Toumbas, Invasion of the giant gravitons from Anti-de Sitter space, JHEP 06 (2000) 008 [hep-th/0003075] [INSPIRE].

[6] M.T. Grisaru, R.C. Myers and O. Tafjord, SUSY and goliath, JHEP 08 (2000) 040 [hep-th/0008015] [INSPIRE].

[7] A. Hashimoto, S. Hirano and N. Itzhaki, Large branes in AdS and their field theory dual, JHEP 08 (2000) 051 [hep-th/0008016] [INSPIRE].

[8] S. Corley, A. Jevicki and S. Ramgoolam, Exact correlators of giant gravitons from dual $N=4$ SYM theory, Adv. Theor. Math. Phys. 5 (2002) 809 [hep-th/0111222] [INSPIRE].

[9] D. Berenstein, A Toy model for the AdS/CFT correspondence, JHEP 07 (2004) 018 [hep-th/0403110] [INSPIRE].

[10] V. Balasubramanian, M. Berkooz, A. Naqvi and M.J. Strassler, Giant gravitons in conformal field theory, JHEP 04 (2002) 034 [hep-th/0107119] [INSPIRE].

[11] N. Beisert, The complete one loop dilatation operator of $N=4$ superYang-Mills theory, Nucl. Phys. B 676 (2004) 3 [hep-th/0307015] [INSPIRE].

[12] R. de Mello Koch and S. Ramgoolam, A double coset ansatz for integrability in AdS/CFT, JHEP 06 (2012) 083 [arXiv: 1204.2153] [INSPIRE].

[13] V. Balasubramanian, D. Berenstein, B. Feng and M.-x. Huang, D-branes in Yang-Mills theory and emergent gauge symmetry, JHEP 03 (2005) 006 [hep-th/0411205] [INSPIRE].

[14] R. de Mello Koch, J. Smolic and M. Smolic, Giant Gravitons - with Strings Attached (I), JHEP 06 (2007) 074 [hep-th/0701066] [INSPIRE].

[15] R. Bhattacharyya, S. Collins and R. de Mello Koch, Exact Multi-Matrix Correlators, JHEP 03 (2008) 044 [arXiv: 0801.2061 ] [INSPIRE].

[16] Y. Kimura and S. Ramgoolam, Branes, anti-branes and brauer algebras in gauge-gravity duality, JHEP 11 (2007) 078 [arXiv: 0709.2158] [INSPIRE].

[17] T.W. Brown, P.J. Heslop and S. Ramgoolam, Diagonal multi-matrix correlators and BPS operators in $N=4$ SYM, JHEP 02 (2008) 030 [arXiv:0711.0176] [INSPIRE]. 
[18] T.W. Brown, P.J. Heslop and S. Ramgoolam, Diagonal free field matrix correlators, global symmetries and giant gravitons, JHEP 04 (2009) 089 [arXiv:0806.1911] [INSPIRE].

[19] Y. Kimura and S. Ramgoolam, Enhanced symmetries of gauge theory and resolving the spectrum of local operators, Phys. Rev. D 78 (2008) 126003 [arXiv:0807.3696] [INSPIRE].

[20] R. Bhattacharyya, R. de Mello Koch and M. Stephanou, Exact Multi-Restricted Schur Polynomial Correlators, JHEP 06 (2008) 101 [arXiv:0805.3025] [INSPIRE].

[21] V. De Comarmond, R. de Mello Koch and K. Jefferies, Surprisingly Simple Spectra, JHEP 02 (2011) 006 [arXiv: 1012.3884] [INSPIRE].

[22] R. de Mello Koch, M. Dessein, D. Giataganas and C. Mathwin, Giant Graviton Oscillators, JHEP 10 (2011) 009 [arXiv:1108.2761] [INSPIRE].

[23] R. de Mello Koch, J. Smolic and M. Smolic, Giant Gravitons - with Strings Attached (II), JHEP 09 (2007) 049 [hep-th/0701067] [INSPIRE].

[24] T.W. Brown, Permutations and the Loop, JHEP 06 (2008) 008 [arXiv:0801.2094] [INSPIRE].

[25] R. de Mello Koch, P. Diaz and N. Nokwara, Restricted Schur Polynomials for Fermions and integrability in the $\mathrm{SU}(2 \mid 3)$ sector, JHEP 03 (2013) 173 [arXiv:1212.5935] [INSPIRE].

[26] D. Berenstein, Giant gravitons: a collective coordinate approach, Phys. Rev. D 87 (2013) 126009 [arXiv: 1301.3519 ] [INSPIRE].

[27] D. Berenstein and E. Dzienkowski, Open spin chains for giant gravitons and relativity, JHEP 08 (2013) 047 [arXiv: 1305.2394] [INSPIRE].

[28] D. Berenstein and E. Dzienkowski, Giant gravitons and the emergence of geometric limits in $\beta$-deformations of $\mathcal{N}=4$ SYM, JHEP 01 (2015) 126 [arXiv:1408.3620] [INSPIRE].

[29] D. Berenstein, On the central charge extension of the $\mathcal{N}=4$ SYM spin chain, JHEP 05 (2015) 129 [arXiv: 1411.5921] [INSPIRE].

[30] R. de Mello Koch, N.H. Tahiridimbisoa and C. Mathwin, Anomalous Dimensions of Heavy Operators from Magnon Energies, JHEP 03 (2016) 156 [arXiv:1506.05224] [INSPIRE].

[31] W. Carlson, R. de Mello Koch and H. Lin, Nonplanar Integrability, JHEP 03 (2011) 105 [arXiv: 1101.5404] [INSPIRE].

[32] R. de Mello Koch, N. Ives and M. Stephanou, On subgroup adapted bases for representations of the symmetric group, J. Phys. A 45 (2012) 135204 [arXiv:1112.4316] [INSPIRE].

[33] R. de Mello Koch and S. Ramgoolam, Strings from Feynman Graph counting: without large N, Phys. Rev. D 85 (2012) 026007 [arXiv:1110.4858] [inSPIRE].

[34] D. Sadri and M.M. Sheikh-Jabbari, Giant hedgehogs: Spikes on giant gravitons, Nucl. Phys. B 687 (2004) 161 [hep-th/0312155] [INSPIRE].

[35] S. de Carvalho, R. de Mello Koch and M. Kim, Central Charges for the Double Coset, JHEP 05 (2020) 007 [arXiv:2001.10181] [INSPIRE].

[36] N. Beisert, The $\mathrm{SU}(2 \mid 3)$ dynamic spin chain, Nucl. Phys. B 682 (2004) 487 [hep-th/0310252] [INSPIRE].

[37] B. Eden, C. Jarczak and E. Sokatchev, A Three-loop test of the dilatation operator in $N=4$ SYM, Nucl. Phys. B 712 (2005) 157 [hep-th/0409009] [INSPIRE]. 
[38] R. de Mello Koch, S. Graham and W. Mabanga, Subleading corrections to the Double Coset Ansatz preserve integrability, JHEP 02 (2014) 079 [arXiv:1312.6230] [INSPIRE].

[39] S. de Carvalho, R. de Mello Koch and A. Larweh Mahu, Anomalous dimensions from boson lattice models, Phys. Rev. D 97 (2018) 126004 [arXiv:1801.02822] [INSPIRE].

[40] R.C. Myers, Dielectric branes, JHEP 12 (1999) 022 [hep-th/9910053] [InSPIRE].

[41] R. de Mello Koch, G. Mashile and N. Park, Emergent Threebrane Lattices, Phys. Rev. D 81 (2010) 106009 [arXiv: 1004.1108] [INSPIRE].

[42] H. Lin, O. Lunin and J.M. Maldacena, Bubbling AdS space and 1/2 BPS geometries, JHEP 10 (2004) 025 [hep-th/0409174] [INSPIRE].

[43] R. de Mello Koch, P. Diaz and H. Soltanpanahi, Non-planar Anomalous Dimensions in the sl(2) Sector, Phys. Lett. B 713 (2012) 509 [arXiv:1111.6385] [INSPIRE].

[44] J.M. Maldacena, S.H. Shenker and D. Stanford, A bound on chaos, JHEP 08 (2016) 106 [arXiv: 1503.01409] [INSPIRE].

[45] A. Kitaev, A simple model of quantum holography. Part 2, talk given at the Entanglement in Strongly-Correlated Quantum Matter, Santa Barbara, California, U.S.A., 6 April-2 July 2015 and online at http://online.kitp.ucsb.edu/online/entangled15/kitaev2.

[46] S. Sachdev and J. Ye, Gapless spin fluid ground state in a random, quantum Heisenberg magnet, Phys. Rev. Lett. 70 (1993) 3339 [cond-mat/9212030] [INSPIRE].

[47] J.M. Maldacena and D. Stanford, Remarks on the Sachdev-Ye-Kitaev model, Phys. Rev. D 94 (2016) 106002 [arXiv: 1604.07818] [INSPIRE].

[48] N. Beisert, The SU(2|2) dynamic S-matrix, Adv. Theor. Math. Phys. 12 (2008) 945 [hep-th/0511082] [INSPIRE].

[49] N. Beisert, The Analytic Bethe Ansatz for a Chain with Centrally Extended su(2|2) Symmetry, J. Stat. Mech. 0701 (2007) P01017 [nlin/0610017] [INSPIRE].

[50] R. de Mello Koch, T.K. Dey, N. Ives and M. Stephanou, Correlators Of Operators with a Large R-charge, JHEP 08 (2009) 083 [arXiv: 0905.2273] [INSPIRE].

[51] R. de Mello Koch, C. Mathwin and H.J.R. Van Zyl, LLM Magnons, JHEP 03 (2016) 110 [arXiv: 1601.06914] [INSPIRE].

[52] R. de Mello Koch, M. Kim and H.J.R. Van Zyl, Integrable Subsectors from Holography, JHEP 05 (2018) 198 [arXiv: 1802.01367] [INSPIRE].

[53] M. Kim and H.J.R. Van Zyl, Semiclassical SL(2) strings on LLM backgrounds, Phys. Lett. B 784 (2018) 62 [arXiv:1805.12460] [INSPIRE].

[54] R. de Mello Koch, J.-H. Huang and L. Tribelhorn, Exciting LLM Geometries, JHEP 07 (2018) 146 [arXiv: 1806. 06586] [INSPIRE].

[55] R. Suzuki, Three-point functions in $\mathcal{N}=4 S Y M$ at finite $N_{c}$ and background independence, JHEP 05 (2020) 118 [arXiv: 2002.07216] [INSPIRE]. 